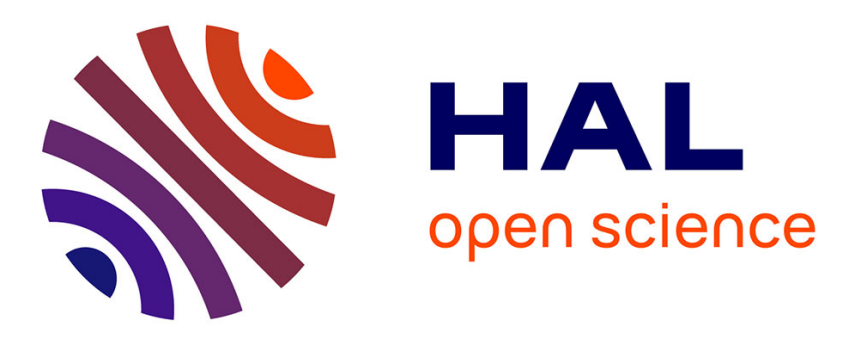

\title{
An allocation rule for dynamic random network formation processes
}

Jean-François Caulier, Michel Grabisch, Agnieszka Rusinowska

\section{To cite this version:}

Jean-François Caulier, Michel Grabisch, Agnieszka Rusinowska. An allocation rule for dynamic random network formation processes. Economic Theory, 2015, 60 (2), pp.283-313. 10.1007/s00199-0150879-6 . halshs-01207823

\section{HAL Id: halshs-01207823 \\ https://shs.hal.science/halshs-01207823}

Submitted on 1 Oct 2015

HAL is a multi-disciplinary open access archive for the deposit and dissemination of scientific research documents, whether they are published or not. The documents may come from teaching and research institutions in France or abroad, or from public or private research centers.
L'archive ouverte pluridisciplinaire HAL, est destinée au dépôt et à la diffusion de documents scientifiques de niveau recherche, publiés ou non, émanant des établissements d'enseignement et de recherche français ou étrangers, des laboratoires publics ou privés. 


\title{
An allocation rule for dynamic random network formation processes ${ }^{\star}$
}

\author{
Jean-François Caulier $^{1}$, Michel Grabisch ${ }^{2 \star \star}$, and Agnieszka Rusinowska ${ }^{3}$ \\ 1 Université Paris I Panthéon-Sorbonne, Centre d'Economie de la Sorbonne \\ jean-francois.caulier@univ-paris1.fr \\ 2 Paris School of Economics, Université Paris I Panthéon-Sorbonne \\ Centre d'Economie de la Sorbonne, 106-112 Bd de l'Hôpital, 75647 Paris Cedex 13, France \\ michel.grabisch@univ-paris1.fr \\ 3 Paris School of Economics - CNRS, Centre d'Economie de la Sorbonne \\ agnieszka.rusinowska@univ-paris1.fr
}

\begin{abstract}
Most allocation rules for network games presented in the literature assume that the network structure is fixed. We put explicit emphasis on the construction of networks and examine the dynamic formation of networks whose evolution across time periods is stochastic. Time-series of networks are studied that describe processes of network formation where links may appear or disappear at any period. Moreover, convergence to an efficient network is not necessarily prescribed. Transitions from one network to another are random and ruled by a stochastic process, typically a Markov chain. We propose the link-based scenario allocation rule for such dynamic random network formation processes and provide its axiomatic characterization. By considering a monotone game and a particular (natural) network formation process we recover the link-based flexible network allocation rule of Jackson (2005a).
\end{abstract}

JEL Classification: C71, D85

Keywords: dynamic networks, network game, link-based allocation rule, Markov chain, characterization

Corresponding author: Michel Grabisch

\section{Introduction}

Interactions can be naturally modeled by networks, and consequently, successfully studied with the support of network theory. Interacting individuals can be viewed as players that are linked in a network and contribute to a total productive value or utility of the network. One of the key questions in a cooperative setting is how to divide between the players the value generated by the network. It means defining an allocation rule that specifies for each member his share of the value of the network. Different proposals - both cooperative and non-cooperative foundations of network allocation rules - have been proposed in the literature. For more detailed surveys of the vast literature on allocation rules we refer, e.g., to Slikker and van den Nouweland (2001), Dutta and Jackson (2003), van den Nouweland (2005), Jackson (2005b, 2008), and also to Section 5 of this paper.

\footnotetext{
* This research is supported by the project DynaMITE (ANR-13-BSH1-0010-01), funded by National Agency for Research (Agence Nationale de la Recherche). The authors thank cordially Matthew Jackson and participants of the Summer Workshop in Economic Theory (SWET13) in Paris, in particular, Bernard Cornet, Anne van den Nouweland and Myrna Wooders for helpful comments. Many thanks are due to the anonymous referees whose insightful comments have permitted to greatly improve the manuscript.

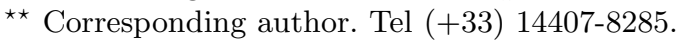


One of the crucial features of real-life social and economic interactions is the fact that they are usually not static. Although dynamic networks, i.e., networks that evolve over time, can be particularly useful for modeling such interactions, insufficient attention is still paid to network dynamics. In particular, most allocation rules for network games usually avoid dynamic aspects of network formation and assume that the network structure is fixed. The key question is therefore how to distribute between players the value generated by a dynamic network. Jackson (2005a) introduced a new class of allocation rules that take into account the potential alternative constructions of the network, by assuming that the efficient network will eventually emerge. He considered the so-called player-based flexible network allocation rule and the link-based flexible network allocation rule. The latter one is denoted here as the LBFN rule. However, in the approach used in Jackson (2005a), even if the allocation is being decided upon when the network is formed or can still be changed, the framework is still static, since the dynamics is just introduced in the fictive construction/decomposition of the structure.

In the present work, we aim at putting explicit emphasis on the construction of networks whose evolution across time periods is stochastic and at studying how to distribute between players the value generated by dynamic networks. The main contribution of the paper is to introduce and characterize axiomatically an allocation rule for dynamic random network formation processes. We are interested in the perspective of assigning values to links rather than players, and consequently, our allocation rule is related to the LBFN rule by Jackson (2005a). We call it the link-based allocation rule for dynamic random network formation processes and denote it as the LBD allocation rule. While the LBFN rule takes the value function into consideration in order to allocate the value, it neglects which network is eventually achieved and how it is actually reached. Our LBD rule does fill in this gap and takes into account both the marginal value of the links that the players are involved in and the scenario, i.e., how active in the network formation process the players are.

We study time-series of networks that describe processes of network formation where several players or links may appear or disappear at any period. Convergence to one of the efficient networks does not necessarily need to be prescribed. One of the basic notions in our framework is the notion of a scenario of network formation processes which is simply a sequence of networks that are observed at subsequent time periods. We restrict our analysis to finite scenarios. A two-network sequence in a scenario is called a transition and is elementary if the two networks differ from each other only by one link. Transitions from one network to another are random and ruled by a stochastic process, typically a Markov chain. A scenario allocation rule assigns to every value function a vector of allocations for every player and every scenario. In order to specify how the value generated by a dynamic process is distributed among players, an allocation rule for dynamic network processes is defined as the expected value over all possible scenarios of the scenario allocation rules. The LBD scenario allocation rule is equal to the sum of the transition allocation rules over the transitions that form the given scenario. We provide an axiomatic characterization of the LBD scenario allocation rule which is based on a set of six natural axioms. We show that for a monotone game, the link-based flexible network allocation rule of Jackson (2005a) coincides with our allocation rule associated to the so-called natural network formation process. In such a process, all scenarios are 
equally probable, we start with the empty network and add one link at each step until we get the complete network.

The remainder of the paper is organized as follows. In Section 2 we recapitulate basic concepts on networks that will be used in the paper. In Section 3 our framework and the link-based allocation rule for dynamic network processes are introduced. In Section 4 we establish the axiomatic characterization for this new allocation rule. In Section 5 we provide a short overview of the related literature. Section 6 presents some concluding remarks. Proofs of the main results and independence of the axioms are presented in Appendix A and Appendix B, respectively.

\section{Preliminaries on networks and allocation rules}

In this section we recall some preliminaries and standard notations concerning networks and allocation rules. Some notations related exclusively to our dynamic model will be introduced in the next section.

Consider a fixed finite set of players $N=\{1, \ldots, n\}$ connected in some network relationship. A network $g$ is a set of pairs $i j$ of players ${ }^{1}$ with $i, j \in N, i \neq j{ }^{2}$ where $i j \in g$ indicates the presence of a link between players $i$ and $j$. Networks under consideration are undirected.

Two particular network relationships among players in $N$ are easily identified: the empty network $g^{\emptyset}$ without any link between players, and the complete network $g^{N}$ which is the set of all possible subsets of $N$ of size 2. Let $G$ be the set of all possible network relationships among players in $N$, i.e., $G=\left\{g \mid g \subseteq g^{N}\right\} .{ }^{3}$ We use the following standard set operations

$$
\begin{gathered}
g \cup g^{\prime}=\left\{i j \mid i j \in g \text { or } i j \in g^{\prime}\right\} \\
g \cap g^{\prime}=\left\{i j \mid i j \in g \text { and } i j \in g^{\prime}\right\} \\
g \backslash g^{\prime}=\left\{i j \mid i j \in g \text { and } i j \notin g^{\prime}\right\}
\end{gathered}
$$

By $g+i j$ we denote the network obtained by adding link $i j$ to an existing network $g$. Similarly, $g-i j$ is the network obtained by deleting link $i j$ from an existing network $g$. For two networks $g$ and $g^{\prime}, g \Delta g^{\prime}$ denotes the symmetric difference, which is the set of links where $g$ and $g^{\prime}$ differ, i.e.,

$$
g \Delta g^{\prime}=\left(g \cup g^{\prime}\right) \backslash\left(g \cap g^{\prime}\right)
$$

Let $L_{i}(g)$ denote the set of links that player $i$ is involved in, and let $\ell(g)$ be the total number of links in $g$, i.e.,

$$
L_{i}(g)=\{i j \mid j \in N \text { and } i j \in g\}, \quad \ell_{i}(g)=\left|L_{i}(g)\right|, \quad \ell(g)=\frac{1}{2} \sum_{i} \ell_{i}(g)
$$

A value function on networks is a mapping $v: G \rightarrow \mathbb{R}$, assigning a real number to any network. This could be for example the benefit or some worth generated by the

\footnotetext{
${ }^{1}$ For convenience we use the shorthand notation $i j$ for the pair $\{i, j\}$.

${ }^{2}$ Loop $i i$ is not a possibility in this setting.

${ }^{3}$ Since $N$ is fixed, in order to simplify the notation, we will use $G$ instead of $G(N)$.
} 
network. For simplicity, we denote $v(\{i j\})$ and $v(\{i j, \ldots, k l\})$ by $v(i j)$ and $v(i j, \ldots, k l)$, respectively. Usually, a pair $(N, v)$ consisting of a player set $N$ and a value function $v \in V$ is called a network game. We denote by $V(N)$ the set of all possible value functions on $N$, or more simply $V$ if $N$ is understood.

A value function $v$ is monotonic if $v\left(g^{\prime}\right) \leq v(g)$ if $g^{\prime} \subseteq g$. Adding links to a network is not detrimental to the value.

Given a value function $v \in V$, the monotonic cover of $v$ is the value function $\hat{v}$ such that $\hat{v}(g)=\max _{g^{\prime} \subseteq g} v\left(g^{\prime}\right)$. The idea is that the players in a given network $g$ may use the available links in any way they want in order to maximize the value generated. Note that $v$ is monotonic if and only if $v=\hat{v}$.

An important example of (monotonic) value functions are unanimity games. For any network $g \in G$, its associated unanimity game $u_{g}$ is defined by

$$
u_{g}\left(g^{\prime}\right)= \begin{cases}1, & \text { if } g^{\prime} \supseteq g \\ 0, & \text { otherwise }\end{cases}
$$

As for TU-games, unanimity games form a basis of the set of network games for a fixed $N$ (simply because the set of links plays exactly the role of $N$ ), and the coefficients in this basis are known as the Möbius inverse or Harsanyi dividends, which we denote by $m^{v}(g), g \subseteq g^{N}$, i.e., it holds

$$
v=\sum_{g \subseteq g^{N}} m^{v}(g) u_{g}
$$

for every $v \in V$, and one has $m^{v}(g)=\sum_{g^{\prime} \subseteq g}(-1)^{\left|g \backslash g^{\prime}\right|} v\left(g^{\prime}\right)$.

A network $g$ is efficient relative to $v$ if it maximizes $v$, i.e., $v(g) \geq v\left(g^{\prime}\right)$ for all $g^{\prime} \in G$.

An allocation rule for a network game $(N, v)$ specifies how the value generated by any network $g$ is allocated among players. Specifically, an allocation rule is a function $Y: G \times V \rightarrow \mathbb{R}^{n}$ such that $\sum_{i} Y_{i}(g, v)=v(g)$ for all $v$ and $g$.

Jackson (2005a) proposes, in particular, the player-based flexible network allocation rule and the link-based flexible network allocation rule. Consider any $v \in V$ and a network $g \in G$. The link-based flexible network allocation rule is defined by

$$
Y_{i}^{L B F N}(g, v)=\frac{v(g)}{\hat{v}\left(g^{N}\right)} \sum_{j \neq i}\left[\sum_{g^{\prime} \subseteq g^{N}-i j} \frac{1}{2}\left(\hat{v}\left(g^{\prime}+i j\right)-\hat{v}\left(g^{\prime}\right)\right)\left(\frac{\ell\left(g^{\prime}\right) !\left(\ell\left(g^{N}\right)-\ell\left(g^{\prime}\right)-1\right) !}{\ell\left(g^{N}\right) !}\right)\right]
$$

Note that if $g$ is efficient, then $v(g)=\hat{v}(g)=\hat{v}\left(g^{N}\right)$. Hence the normalization factor $\frac{v(g)}{\hat{v}\left(g^{N}\right)}$ disappears.

Let us make some comments about this rule, which will motivate the construction of our new rule. As far as we know, the LBFN rule is one of the few examples of an allocation rule trying to take into account some dynamics of the network formation. It is called "flexible" because it is considered that the network $g$ under consideration, which is not necessarily efficient, should evolve towards some efficient network. However, note that the way this evolution is realized is ignored by the rule, as well as the true final efficient network, which is not necessarily $g^{N}$. Also, remark that $g$ itself appears in the formula only as a normalization factor, but not directly in the computation. Lastly, we 
observe that it is $\hat{v}$ instead of $v$ which is used in the formula. However, infinitely many games have the same monotonic cover, hence the information conveyed by $v$ is partially ignored in the computation.

Based on these observations, we would like to propose an allocation rule which assumes that the network is not fixed and does not suppose that eventually the complete network or an efficient network will form. Rather, we consider that the evolution is free. Second, this rule should take fully into account the information contained in $v$, as well as the evolution of the network from the beginning to the end. As a conclusion, instead of $Y(g, v)$ or $Y\left(g^{N}, \hat{v}\right)$ we should have $Y(\mathcal{G}, v)$ where $\mathcal{G}$ is a "scenario" of network evolution, or better $Y(U, v)$, where $U$ is some stochastic process ruling the evolution of the network (see Subsection 3.1 for the formal definitions of $\mathcal{G}$ and $U$ ). This motivates the construction we propose in the following sections.

\section{Scenarios and dynamic network processes}

\subsection{General description}

We assume that networks are evolving along time, at discrete time steps. The evolution is ruled by some process, whose precise nature is outside the scope of this paper, and as it will be seen, irrelevant to our study of an allocation rule. Formally, a dynamic network process is a discrete stochastic process $U$, where $U\left(s_{t}, g_{t}\right)$ is the probability distribution over $G$ of $g_{t+1}$, the network at time step $t+1$, given the state of the system $s_{t}$ at time $t$ and the current network $g_{t}$. A simple and widely studied example of such processes is the discrete Markov process, described briefly at the end of this subsection. Strategic models of network formation, where players have incentives to create or delete links, can in general be reduced to this general view of dynamic network processes.

A particular instance of the evolution of a network is called a scenario. Formally, a scenario is a sequence of networks

$$
\mathcal{G}=g_{0}, g_{1}, g_{2}, g_{3} \ldots
$$

where $g_{t} \in G$ for $t=0,1,2, \ldots$ with the meaning that $g_{t}$ is the network observed at time period $t$. Note that scenarios need not finish at the complete network $g^{N}$, and a given network may appear several times in a scenario. A normal scenario is such that $g_{0}=g^{\emptyset}$.

We limit ourselves to scenarios of finite length, partly because of simplicity, but essentially because the computation of an allocation to players necessarily involves a finite horizon $T$ for the observation of the evolution. We denote by $\mathfrak{S}(T)$ the (finite) set of all scenarios of length $T, T \in \mathbb{N}^{4}$

The dynamic network process being given, it is possible to compute the probability $P(\mathcal{G})$ of any scenario $\mathcal{G} \in \mathfrak{S}(T)$. As it will become apparent in the sequel, only these probabilities matter, so that we can identify the set of dynamic network processes with the set of probability distributions over $\mathfrak{S}(T)$, which we denote by $\mathfrak{P}(T)$.

Any subsequence $g_{t}, g_{t+1}$ of subsequent networks in $\mathcal{G}$ is called a transition and will be denoted by $g_{t} \rightarrow g_{t+1}$. A transition $g_{t} \rightarrow g_{t+1}$ is elementary if $g_{t+1}$ differs from $g_{t}$ only by one link.

\footnotetext{
${ }^{4}$ For a discussion of a possibility to consider infinite scenarios produced by Markov processes, see Faigle and Grabisch (2012).
} 
Example 1 Consider a small group consisting of 3 researchers in a lab: Agnieszka $(A)$, Jean-François $(J)$ and Michel $(M)$, i.e., we have $N=\{A, J, M\}^{5}$. The lab wants to promote collaboration among its members by announcing regular calls for 2-person projects. A link between any two researchers means submitting a project by these two researchers for getting a grant. An example of a normal finite scenario is

$$
\mathcal{G}=g^{\emptyset},\{A M\},\{A J, J M\}, g^{N},\{A M\},\{A J, A M\}
$$

where each network appearing in this scenario corresponds to the set of the projects submitted for a given call. Consequently, in the scenario $\mathcal{G}$, first Agnieszka and Michel decide to submit a project, but they do not resubmit anything for the next call, where two other projects are submitted: one by Agnieszka and Jean-François, and another one by Jean-François and Michel. Then, at the next call, every pair submits a project. Next, only Agnieszka and Michel submit a project, and finally for the last call, Agnieszka and Jean-François as well as Agnieszka and Michel submit two projects.

In the scenario $\mathcal{G}$, three transitions are elementary, i.e.,

$$
g^{\emptyset} \rightarrow\{A M\}, \quad\{A J, J M\} \rightarrow g^{N}, \quad\{A M\} \rightarrow\{A J, A M\}
$$

and the remaining two transitions are not elementary, i.e.,

$$
\{A M\} \rightarrow\{A J, J M\}, \quad g^{N} \rightarrow\{A M\}
$$

A value function $v$ can be defined, where $v(g)$ depends on the CV's of the researchers involved and the quality of their cooperation. We can assume that $v\left(g^{\emptyset}\right)=0$. Figure 1 presents an example of such a value function $v$.
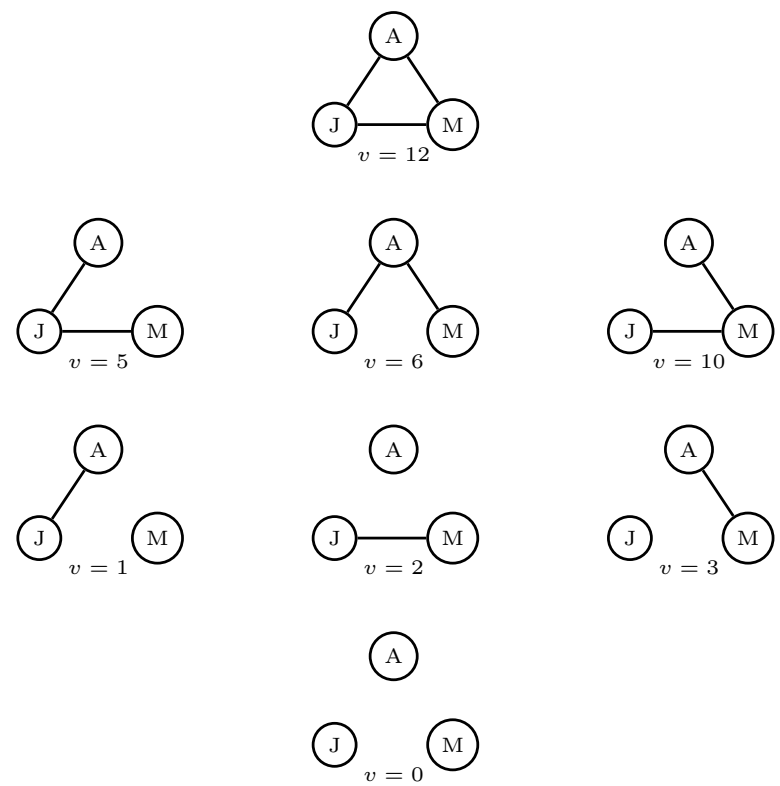

Fig. 1. The 8 possible networks with their value.

\footnotetext{
${ }^{5}$ Any resemblance with existing persons is purely accidental.
} 
Let us apply the link-based flexible network allocation rule (2) to this example. We note that the efficient network is the complete network, and that $v$ is monotone, so that it coincides with $\hat{v}$. We are therefore in a simple case for the computation of the LBFN rule, and most of the drawbacks we pointed out in the foregoing section disappear. We find the following allocation:

$$
Y_{A}^{L B F N}=3.75, \quad Y_{M}^{L B F N}=5, \quad Y_{J}^{L B F N}=3.25 .
$$

We observe that $M$ gets the highest reward, then $A$ and $J$. This allocation well reflects the value function $v$, in the sense that the order obtained corresponds to the order implied by the marginal values of the links where $A, J$ and $M$ are involved. One may say that this result is therefore satisfactory, however, it does not reflect the scenario that has actually realized. Indeed, one can check that the researchers are not equally active in the scenario: counting +1 whenever a researcher becomes active (i.e., participates to a project to which he/she did not participate at previous step) and -1 whenever he/she becomes inactive, we find the following counts: +2 for Agnieszka, and +1 for Michel and Jean-François. Somehow, these "activity counts" should be reflected by the allocation, which is not the case for the LBFN rule. The example shows that a rule taking both aspects (a kind of marginal value and the scenario) into account is needed.

Among the stochastic processes, of particular interest are the stationary Markov processes, where the probability of transition from one network to another depends only on the current network instead of the whole history (i.e., the state $s$ of the system is simply the current network), and these probabilities are independent of time. Denoting by $p_{g g^{\prime}}$ the probability of transition from $g$ to $g^{\prime}$, it follows that the probability of occurrence of a finite scenario $\mathcal{G}=g_{0}, g_{1}, g_{2}, \ldots, g_{T}$ is given by

$$
P(\mathcal{G})=\operatorname{Pr}\left(g_{0}\right) \prod_{t=1}^{T} p_{g_{t-1} g_{t}}
$$

where $\operatorname{Pr}\left(g_{0}\right)$ is the probability of occurrence of the initial state $g_{0}$. Note that if the process converges to an absorbing network $g$ at time $T^{\prime}$, we have $p_{g g^{\prime}}=0$ for all $g^{\prime} \neq g$ and $p_{g g}=1$, so that the corresponding scenario, if observed on a horizon $T \geq T^{\prime}$, has the same probability for any $T \geq T^{\prime}$. In this sense, all scenarios corresponding to a convergence of the process at some time $T^{\prime} \leq T$ are present in $\mathfrak{S}(T)$.

\subsection{The link-based allocation rule for dynamic network processes}

Next, we will introduce our allocation rule for the dynamic network process described above. First, we start with general definitions.

Definition 1 Let $V$ be the set of value functions and $\mathfrak{S}$ be the set of finite scenarios. A

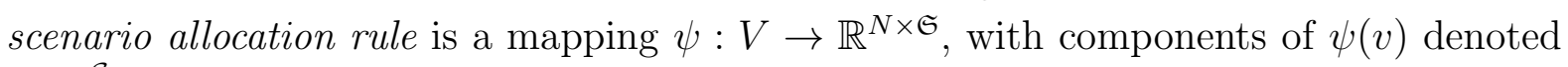
by $\psi_{i}^{\mathcal{G}}$ for player $i$ and scenario $\mathcal{G}$.

Definition 2 Let $\psi$ be a scenario allocation rule. The allocation rule for dynamic network processes on a finite horizon $T$ induced by $\psi$ is a mapping $\Psi: V \times \mathfrak{P}(T) \rightarrow \mathbb{R}^{n}$ which 
assigns to every value function and probability distribution the expected value over all possible scenarios of the scenario allocation rules, i.e.,

$$
\Psi(v, P):=\sum_{\mathcal{G} \in \mathfrak{S}(T)} P(\mathcal{G}) \psi^{\mathcal{G}}(v)
$$

where $P(\mathcal{G})$ is the probability of occurrence of scenario $\mathcal{G}$, according to the probability distribution $P \in \mathfrak{P}(T)$.

We introduce and characterize the so-called link-based allocation rule for dynamic network processes, denoted by $\Phi$ and abbreviated by the $L B D$ (allocation) rule (LBD = link-based dynamic), which is induced by $\phi^{\mathcal{G}}$, the $L B D$ scenario allocation rule. We give first an informal definition of the rule limited to transitions:

- If the transition $g \rightarrow g^{\prime}$ is elementary (i.e., $g^{\prime}$ differs from $g$ only by one link, say $i j$ ), then

$$
\phi_{i}^{g \rightarrow g^{\prime}}=\phi_{j}^{g \rightarrow g^{\prime}}=\frac{1}{2}\left(v\left(g^{\prime}\right)-v(g)\right)
$$

and for all other players $\phi_{k}^{g \rightarrow g^{\prime}}=0$.

- If the transition is not elementary, we take the average over all possible shortest paths formed of elementary transitions from $g$ to $g^{\prime}$.

In order to get a formal definition, we introduce some additional concepts and notations.

Definition 3 A player $i$ is adjacent to graph $g$ if he is adjacent to some link in $g$, i.e., there exists $j \in N$ such that $i j \in g$. We denote it by $i \rightarrow g$ or $g<i$. Similarly, a link $i j$ is adjacent to a graph $g$ if either $i \rightarrow g$ or $j \rightarrow g$. We denote it by $i j \rightarrow g$ or $g \leftarrow i j$.

Consider a transition $g \rightarrow g^{\prime}$. Links in $g^{\prime}$ but not in $g$ are called entering links, while those in $g$ but not more in $g^{\prime}$ are called leaving links. In order to obtain compact formulations, we introduce the signed version of links and set of links. Considering a transition $g \rightarrow g^{\prime}$, entering links $\lambda \in g^{\prime} \backslash g$ have a positive sign, while leaving links have a negative sign. The signed version of a link $\lambda$ is denoted by $\varepsilon \lambda$, with $\varepsilon=-$ or + depending whether $\lambda$ is leaving or entering. Similarly, if $h$ is a set of links (graph), $\varepsilon h$ is the set of signed links ${ }^{6}$. As a consequence, writing $g+\varepsilon i j$ means $g+i j$ if $\varepsilon=+$ (entering) and $g-i j$ if $\varepsilon=-$ (leaving). Similarly, $g+\varepsilon h$ stands for the less readable $\left(g \cup h^{+}\right) \backslash h^{-}$with $h^{+}$(resp. $h^{-}$) the set of entering (resp. leaving) links in $h$. Note that the "+" operation acts like the usual addition, e.g., $g+i j-i j=g$, except that it is idempotent: $g+i j+i j=g+i j$, $g-i j-i j=g-i j$. Also, note that $g-\varepsilon i j=g+i j$ if $\varepsilon=-$.

In summary, entering and leaving links form the set $g \Delta g^{\prime}$, which could be called the set of active links in transition $g \rightarrow g^{\prime}$. Accordingly, one can define active players.

Definition 4 A player $i$ is said to be active in a transition $g \rightarrow g^{\prime}$ if he is adjacent to $g \Delta g^{\prime}$ (in symbols: $i \rightarrow g \Delta g^{\prime}$ ), otherwise $i$ is said to be inactive in the transition $g \rightarrow g^{\prime}$.

Under these conventions we can introduce the following definition:

\footnotetext{
${ }^{6}$ We are conscious that the notation is ambiguous, but it should cause no problem since sets of links are denoted by $g, g^{\prime}, h, h^{\prime}$, etc.
} 
Definition 5 The link-based allocation rule for dynamic network processes on a finite horizon $T$ (the $L B D$ allocation rule) $\Phi$ is defined as

$$
\Phi(v, P):=\sum_{\mathcal{G} \in \mathfrak{S}(T)} P(\mathcal{G}) \phi^{\mathcal{G}}(v)
$$

and $\phi^{\mathcal{G}}$ is the $L B D$ scenario allocation rule given by

$$
\phi^{\mathcal{G}}(v)=\sum_{k=0}^{T-1} \phi^{g_{k} \rightarrow g_{k+1}}(v)
$$

with $\mathcal{G}=g_{0}, g_{1}, g_{2}, \ldots, g_{T}$ and

$$
\phi_{i}^{g \rightarrow g^{\prime}}(v)= \begin{cases}\frac{1}{2\left|g \Delta g^{\prime}\right| !} \sum_{\sigma} \sum_{i k \in g \Delta g^{\prime}}\left(v\left(g+\varepsilon h_{\sigma}^{i k}\right)-v\left(g+\varepsilon h_{\sigma}^{i k}-\varepsilon i k\right)\right), & \text { if } i \rightarrow g \Delta g^{\prime} \\ 0, & \text { otherwise }\end{cases}
$$

where $\sigma$ is any permutation on the set of links $g \Delta g^{\prime}=\left\{\lambda_{1}, \ldots, \lambda_{\left|g \Delta g^{\prime}\right|}\right\}$, and $h_{\sigma}^{i k}$ is the first set in the sequence $\left\{\lambda_{\sigma(1)}\right\},\left\{\lambda_{\sigma(1)}, \lambda_{\sigma(2)}\right\}, \ldots, g \Delta g^{\prime}$ containing $i k$.

Note that inactive players receive 0 .

Remark 1 It is important to note that the two levels (the allocation rule level and the scenario allocation rule level) work independently. That is, on the allocation rule level, the transitions between networks obey a stochastic process, which determines the probability of transitions of a network $g$ into another network $g^{\prime}$. This stochastic process typically results of the behavior of the players and, in a sense, comes from the players taken as individuals. By contrast, on the scenario rule level, for a given transition $g \rightarrow g^{\prime}$, one has to define in a proper way how the benefit/loss of the transition (i.e., $v\left(g^{\prime}\right)-v(g)$ ) is shared among the players who are active in the transition. This could be seen as a basic rule imposed by the "network", i.e., by the players considered as a society, and which therefore operates on a different level. If one imposes symmetry in the sharing (no player has a special advantage, only $v$ and the structure of the network matter), our axiomatization shows that we are led to compute, for a nonelementary transition, the (unweighted) average over all possibilities of starting from $g$ and arriving at $g^{\prime}$ by elementary transitions, regardless of the probabilities of those elementary transitions stemming from the stochastic process.

A first result is that we can recover the link-based flexible network allocation rule of Jackson (2005a) by considering a special process, called the natural process.

Definition 6 The natural process is a process of network formation defined by:

- the process starts with the empty network, i.e., $g_{0}=g^{\emptyset}$

- we add one link at each step until we obtain the complete graph $g^{N}$

- all scenarios are of length $\eta$ and are equally probable, i.e., $P(\mathcal{G})=\frac{1}{\eta !}$ for each $\mathcal{G} \in \mathfrak{S}(\eta)$, where $\eta=\left(\begin{array}{l}n \\ 2\end{array}\right)$.

Proposition 1 Assume $v$ is a monotone game. Then for $g=g^{N}$, the $Y^{L B F N}$ rule coincides with $\Phi$ associated to the natural process. 
Proof: When $v$ is monotone, the $Y^{L B F N}$ rule reduces to

$$
Y_{i}^{L B F N}\left(g^{N}, v\right)=\sum_{j \neq i}\left[\sum_{g^{\prime} \subseteq g^{N}-i j} \frac{1}{2}\left(v\left(g^{\prime}+i j\right)-v\left(g^{\prime}\right)\right)\left(\frac{\ell\left(g^{\prime}\right) !\left(\eta !-\ell\left(g^{\prime}\right)-1\right) !}{\eta !}\right)\right]
$$

Take player $i$ and count his contribution $\Delta_{i}$ in each scenario. Consider scenario $\mathcal{G}$. Since $g^{N}$ is complete, player $i$ has degree $n-1$, and therefore $\Delta_{i}$ is non null in exactly $n-1$ transitions of the scenario where links $i j$ are added, $j=1, \ldots, i-1, i+1, \ldots, n$. When the link $i j$ is added, we find

$$
\Delta_{i}=\frac{1}{2}\left(v\left(g^{\prime}+i j\right)-v\left(g^{\prime}\right)\right)
$$

where $g^{\prime}$ is the last graph without $i j$ in $\mathcal{G}$. For a fixed $g^{\prime}$, there are $\ell\left(g^{\prime}\right)$ ! paths from the empty graph to $g^{\prime}$, and $\left(\eta-\ell\left(g^{\prime}\right)-1\right)$ ! paths from $g^{\prime}+i j$ to the complete graph $g^{N}$, hence the result.

Example 1 (ctd.) Consider the scenario $\mathcal{G}$ given in Example 1. By virtue of (5), we have

$$
\phi^{\mathcal{G}}(v)=\phi^{g^{\emptyset} \rightarrow\{A M\}}(v)+\phi^{\{A M\} \rightarrow\{A J, J M\}}(v)+\phi^{\{A J, J M\} \rightarrow g^{N}}(v)+\phi^{g^{N} \rightarrow\{A M\}}(v)+\phi^{\{A M\} \rightarrow\{A J, A M\}}(v)
$$

The symmetric differences and players adjacent to them are the following:

$$
\begin{gathered}
g^{\emptyset} \Delta\{A M\}=\{A M\}, \quad\{A M\} \Delta\{A J, J M\}=g^{N}, \quad\{A J, J M\} \Delta g^{N}=\{A M\} \\
g^{N} \Delta\{A M\}=\{A J, J M\}, \quad\{A M\} \Delta\{A J, A M\}=\{A J\} \\
A, M \rightarrow\{A M\}, \quad A, J, M \rightarrow g^{N} \\
A, J, M \rightarrow\{A J, J M\}, \quad A, J \rightarrow\{A J\}
\end{gathered}
$$

Let us apply (6) to the example. First, for the elementary transitions we have

$$
\begin{gathered}
\phi_{A}^{g^{\emptyset} \rightarrow\{A M\}}(v)=\phi_{M}^{g^{\emptyset} \rightarrow\{A M\}}(v)=\frac{v(A M)}{2}, \quad \phi_{J}^{g^{\emptyset} \rightarrow\{A M\}}(v)=0 \\
\phi_{A}^{\{A J, J M\} \rightarrow g^{N}}(v)=\phi_{M}^{\{A J, J M\} \rightarrow g^{N}}(v)=\frac{v\left(g^{N}\right)-v(A J, J M)}{2}, \quad \phi_{J}^{\{A J, J M\} \rightarrow g^{N}}(v)=0 \\
\phi_{A}^{\{A M\} \rightarrow\{A J, A M\}}(v)=\phi_{J}^{\{A M\} \rightarrow\{A J, A M\}}(v)=\frac{v(A J, A M)-v(A M)}{2}, \quad \phi_{M}^{\{A M\} \rightarrow\{A J, A M\}}(v)=0
\end{gathered}
$$

Consider now the (not elementary) transition $\{A M\} \rightarrow\{A J, J M\}$. There are six shortest sequences of elementary transitions between $\{A M\}$ and $\{A J, J M\}$, as presented in Figure 2. The red/green/blue arrows in this figure indicate the transitions where $A / J / M$ is active.

We have then

$$
\phi_{A}^{\{A M\} \rightarrow\{A J, J M\}}(v)=\frac{1}{12}[v(A J)-v(A M)-v(A M)+v(A J, J M)-v(J M)+v(A J)-v(A M)+
$$




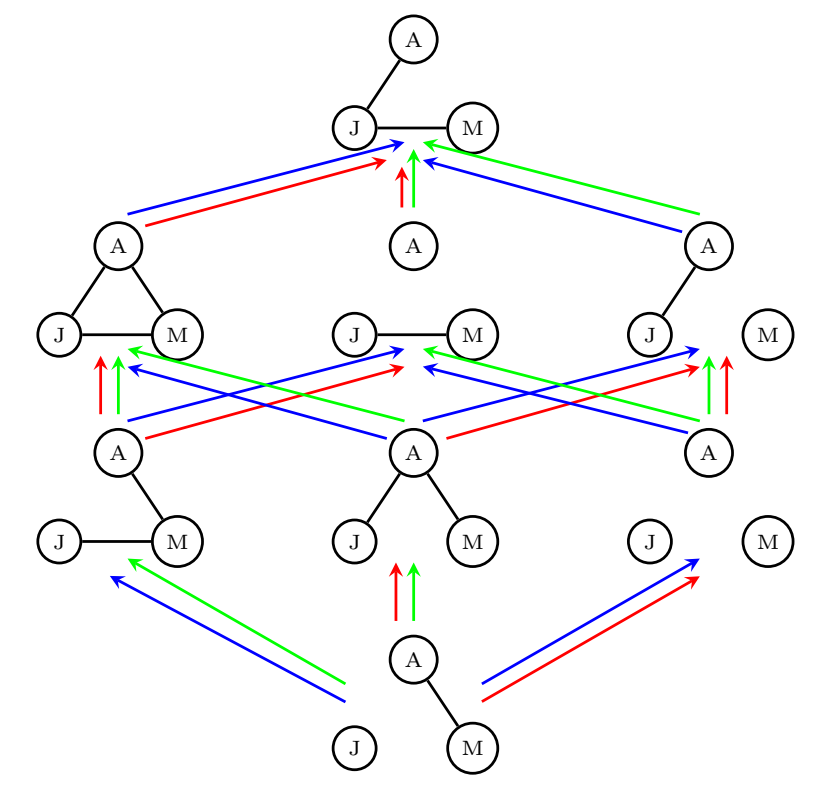

Fig. 2. The shortest sequences of elementary transitions between $\{A M\}$ and $\{A J, J M\}$ and their active players (red/green/blue arrows indicate the transitions where $A / J / M$ is active).

$$
\begin{aligned}
& \left.v(A J, A M)-v(A M)+v(A J, J M)-v\left(g^{N}\right)+v(A J, J M)-v(A M, J M)+v(A J, J M)-v(A M, J M)\right] \\
& =\frac{1}{12}\left[v(A J, A M)-v(J M)-v\left(g^{N}\right)+2(v(A J)-v(A M, J M))+4(v(A J, J M)-v(A M))\right] \\
& \phi_{J}^{\{A M\} \rightarrow\{A J, J M\}}(v)=\frac{1}{12}[v(A J, J M)+v(A J, J M)+v(A J, A M)-v(A M)+v(A J, J M)-v(A J) \\
& \left.+v\left(g^{N}\right)-v(A M)+v(A M, J M)-v(A M)+v(A J, J M)-v(J M)+v\left(g^{N}\right)-v(A M)\right]= \\
& =\frac{1}{12}\left[2 v\left(g^{N}\right)+4 v(A J, J M)+v(A J, A M)+v(A M, J M)-v(J M)-v(A J)-4 v(A M)\right] \\
& \quad \phi_{M}^{\{A M\} \rightarrow\{A J, J M\}}(v)=\frac{1}{12}[-v(A M)+v(A J, J M)-v(A J)+2(v(J M)-v(A M))+ \\
& \left.\quad+2(v(A J, J M)-v(A J, A M))+v(A M, J M)-v(A M)+v(A J, J M)-v\left(g^{N}\right)\right]= \\
& =\frac{1}{12}\left[v(A M, J M)+2(v(J M)-v(A J, A M))+4(v(A J, J M)-v(A M))-v\left(g^{N}\right)-v(A J)\right]
\end{aligned}
$$

In a similar way we can calculate $\phi_{i}^{g^{N} \rightarrow\{A M\}}(v)$ for $i \in N$. We have then

$$
\begin{aligned}
\phi_{A}^{g^{N} \rightarrow\{A M\}}(v)= & \frac{1}{4}\left[-v\left(g^{N}\right)+v(A M, J M)-v(A J, A M)+v(A M)\right] \\
& \phi_{J}^{g^{N} \rightarrow\{A M\}}(v)=\frac{1}{2}\left[v(A M)-v\left(g^{N}\right)\right] \\
\phi_{M}^{g^{N} \rightarrow\{A M\}}(v)= & \left.\frac{1}{4}\left[-v(A M, J M)+v(A M)-v\left(g^{N}\right)\right)+v(A J, A M)\right]
\end{aligned}
$$


Note that for our scenario $\mathcal{G}$ we have $\phi_{A}^{\mathcal{G}}+\phi_{J}^{\mathcal{G}}+\phi_{M}^{\mathcal{G}}=v(A J, A M)$. In the next section we will show that the LBD scenario allocation rule is indeed efficient.

With the specific values $v(g)$ for all $g$ given in Figure 1 and scenario $\mathcal{G}$, the LBD scenario allocation rule gives the following distribution:

$$
\phi_{A}^{\mathcal{G}}=3.75, \quad \phi_{M}^{\mathcal{G}}=1.5, \quad \phi_{J}^{\mathcal{G}}=0.75 .
$$

The first difference between the LBD scenario allocation rule and the link-based flexible network allocation rule concerns the value distributed. The former one distributes the difference of value between the end network and the first in the scenario (in this case, 6), whereas the latter distributes the value of the efficient network under $v$ (which is 12). The second striking difference between both allocation rules is that despite the experience and the central role of Michel reflected by the value function $v$, the LBD scenario allocation rule gives a larger share to Agnieszka, which is, as we have seen, the most active player under the scenario $\mathcal{G}$ : her activity count is +2 , while Michel and Jean-François have only +1 . The link-based flexible allocation $(\mathrm{LBFN})$ rule takes the value function into consideration in order to allocate the value but neglects which network is eventually achieved and how it is actually reached. This can be clearly explained by Proposition 1 . Indeed, the LBFN rule supposes implicitly that the natural process has realized, i.e., all scenarios from the empty network to the complete network with elementary transitions are considered, which results in an equal activity count for all agents. The advantage of the LBD rule is that only specific scenarios, without any restriction, can be considered.

\section{Axiomatization of the LBD scenario allocation rule}

Next, we provide an axiomatic characterization of the LBD scenario allocation rule. As introduced in Definition $1, \psi^{\mathcal{G}}$ denotes a scenario allocation rule for scenario $\mathcal{G}$.

Definition 7 Two sequences $\mathcal{G}=g_{0}, \ldots, g_{q}, \mathcal{G}^{\prime}=g_{0}^{\prime}, \ldots, g_{r}^{\prime}$ are said to be concatenable if $g_{q}=g_{0}^{\prime}$, in which case their concatenation is the sequence

$$
\mathcal{G} \oplus \mathcal{G}^{\prime}:=g_{0}, \ldots, g_{q}, g_{1}^{\prime}, \ldots, g_{r}^{\prime} .
$$

Concatenation $(\mathrm{C})$ : Let $\mathcal{G}, \mathcal{G}^{\prime}$ be two concatenable sequences. Then

$$
\psi^{\mathcal{G} \oplus \mathcal{G}^{\prime}}=\psi^{\mathcal{G}}+\psi^{\mathcal{G}^{\prime}} .
$$

Axiom (C) allows to restrict our attention to transitions. Indeed,

$$
\psi^{\mathcal{G}}=\sum_{k=0}^{T-1} \psi^{g_{k} \rightarrow g_{k+1}}
$$

holds for every sequence $\mathcal{G}=g_{0}, g_{1}, \ldots, g_{T}$.

Basically, the axiom says that it is equivalent to pay allocation to players on an 'every day basis' (at each transition), or for a given long period of time (the whole scenario). In a scenario with networks evolving over time, the activity of a player in every transition of the scenario, i.e., in every stage of the network evolution, counts for the allocation to the player received in the whole dynamic process. 
Efficiency (E): For any finite scenario $\mathcal{G}=g_{0}, g_{1}, \ldots, g_{T}$ it holds $\sum_{i \in N} \psi_{i}^{\mathcal{G}}=$ $v\left(g_{T}\right)-v\left(g_{0}\right)$.

We take the perspective that for a scenario the difference between the values of the last and the first network in that scenario has to be allocated. Every transition in a given scenario is important as it is part of the network evolution. A natural way to evaluate the payoff of a transition is to consider the difference between the new and the previous network of the transition. Taking the sum of these difference payoffs over all transitions is a collapsing sum that leads to the difference between the value of the last and the first network. An alternative perspective could be to take the sum over all networks of the scenario, which would mean that the accumulated values of the networks is meaningful. Our view is however more on the dynamic side, as we think that only the variation is meaningful.

Note that our efficiency axiom is not related to the concept of network efficiency or Pareto efficiency, as (E) does not involve a comparison of networks, but it concerns the value allocated among players without wastage.

Next we impose the linearity axiom, which is very common in the literature of allocation rules and values of games.

Linearity for transitions (L): $v \mapsto \psi^{g \rightarrow g^{\prime}}(v)$ is a linear operator for any transition $g \rightarrow g^{\prime}$.

Linearity for transitions states that it is sufficient to study allocations on transitions on a linear basis for $V$, where $V$ denotes the set of all possible value functions.

Inactive player (IP): If $i$ is inactive in $g \rightarrow g^{\prime}$, then $\psi_{i}^{g \rightarrow g^{\prime}}(v)=0$ for any $v$.

By Definition 4, inactive players in a transition do not see any change in their links, and therefore are not concerned in this transition. It is then natural that they receive no allocation.

Definition 8 A link $i j$ is null for $v$ if $v(g+i j)=v(g)$ for every $g \not \supset i j$.

For any graph $g$ and game $v$, the graph $g^{*}$ is the graph where all links which are null for $v$ have been deleted.

It should be remarked that a link is active or inactive, depending on the transition $g \rightarrow g^{\prime}$ (see Definition 4), and it is null or nonnull, depending on the value function $v$. These are therefore two independent notions, and according to our notation, $\left(g \Delta g^{\prime}\right)^{*}$ denotes the graph of all active and nonnull links in the transition $g \rightarrow g^{\prime}$, relative to the value function $v$. This graph will play a central role in the next axioms.

Definition 9 Two distinct links $i j, k l$ are symmetric for $v$ if for every graph $g$ not containing them, it holds $v(g+i j)=v(g+k l)$.

Degree axiom (D): Consider a transition $g \rightarrow g^{\prime}$ and a game $v$. If all links in $\left(g^{\prime} \backslash g\right)^{*}$ are pairwise symmetric for $v$, then for all players $i$ not adjacent to some link in $\left(g \backslash g^{\prime}\right)^{*}$, the allocation given to $i$ is proportional to its degree in $\left(g \Delta g^{\prime}\right)^{*}$ :

$$
\psi_{i}^{g \rightarrow g^{\prime}}(v)=\alpha \ell_{i}\left(\left(g \Delta g^{\prime}\right)^{*}\right),
$$

for some $\alpha \in \mathbb{R}$ (and similarly when inverting "entering" and "leaving"). 
Essentially, the axiom says that, first, null links have no contribution in the allocation to players, and second, that in a situation where all entering links have the same effect on the value function (symmetry), every player who does not have leaving links should receive an amount proportional to the number of its entering links (and similarly for leaving links) $)^{7} .8$

Note that from the logical point of view, the condition "If all links in $\left(g^{\prime} \backslash g\right)^{*}$ are pairwise symmetric" is vacuously satisfied when there is no nonnull entering links or only one (and similarly for leaving links). Hence, the axiom covers a wide range of situations. In particular, a player with no nonnull links would receive 0 , as its degree is 0 . Also, if there is only one nonnull entering (or leaving) link $i j$ and that $i, j$ are not adjacent to leaving links, then $i$ and $j$ receive the same allocation.

Lastly, note that the above axiom does not imply the (IP) axiom. Indeed, a player who is inactive in a transition $g \rightarrow g^{\prime}$ receives 0 , even if the links in $g^{\prime} \backslash g$ are not symmetric.

The axiom says nothing for players adjacent to both entering and leaving links. This is the target of the next axiom, and in order to introduce it, an additional property of links is needed.

Definition 10 Two distinct links $i j, k l$ are antisymmetric for $v$ if $v(g+i j+k l)=v(g)$ for every graph $g$ not containing them.

Remark 2 Observe from the definitions that if $\lambda, \lambda^{\prime}$ are antisymmetric and $\lambda^{\prime}, \lambda^{\prime \prime}$ are symmetric, then $\lambda, \lambda^{\prime \prime}$ are antisymmetric as well.

Antisymmetry for entering/leaving links (ASEL): Consider a transition $g \rightarrow g^{\prime}$ and a game $v$. If all links in $\left(g^{\prime} \backslash g\right)^{*}$ are pairwise symmetric for $v$, as well as all links in $\left(g \backslash g^{\prime}\right)^{*}$, and if any pair of links $\lambda \in\left(g^{\prime} \backslash g\right)^{*}, \lambda^{\prime} \in\left(g \backslash g^{\prime}\right)^{*}$ are antisymmetric, then for any player $i \rightarrow g \Delta g^{\prime}$, the allocation given to $i$ is proportional to its degree in $\left(g \Delta g^{\prime}\right)^{*}$ :

$$
\psi_{i}^{g \rightarrow g^{\prime}}(v)=\alpha \ell_{i}\left(\left(g \Delta g^{\prime}\right)^{*}\right)
$$

for some $\alpha \in \mathbb{R}$.

As for (D), null links have no impact on the allocation to players, and the axiom describes a situation where entering links have the same effect on the value function, and similarly for leaving links. The new aspect described by the axiom is how to compare the contribution of leaving and entering links. From Definition 10, two antisymmetric links $i j, k l$ which enter the network annihilate each other, which can be interpreted by saying that they bring the same contribution but of opposite sign, say $+\delta,-\delta$ respectively. Therefore, if we interpret a leaving link as a loss and an entering link as a gain, it turns out that if $i j$ is leaving and $k l$ is entering, their contribution in the transition $g \rightarrow g^{\prime}$ becomes equal: $-(+\delta)=-\delta$ (and similarly if $i j$ is entering and $k l$ is leaving).

\footnotetext{
7 The proportionality constant depends on $v$. It may be negative or even equal to 0 (see the expression of $K$ given by (8) in the proof of Proposition 2).

${ }^{8}$ One may wish to distinguish in two different axioms the fact that null links have no contribution and the symmetry aspect. We would have then, say a null link axiom (NL), saying that if $i j$ is null for $v$, then $\psi^{g \rightarrow g^{\prime}}(v)=\psi^{g \backslash i j \rightarrow g^{\prime} \backslash i j}(v)$, and the modified degree axiom (D') with $g \Delta g^{\prime}$ replacing $\left(g \Delta g^{\prime}\right)^{*}$.
} 
Note that (ASEL) does not imply the (D) axiom, because (D) can be applied to a transition $g \rightarrow g^{\prime}$ having both entering links and leaving links, where entering links are pairwise symmetric, but not necessarily those which are leaving.

Lastly, due to Remark 2, observe that one can replace in the above axiom "any pair of links $\lambda \in\left(g^{\prime} \backslash g\right)^{*}, \lambda^{\prime} \in\left(g \backslash g^{\prime}\right)^{*}$ are antisymmetric" by "there exists a pair of links $\lambda \in\left(g^{\prime} \backslash g\right)^{*}, \lambda^{\prime} \in\left(g \backslash g^{\prime}\right)^{*}$ which are antisymmetric".

We illustrate axioms (IP), (D) and (ASEL) with the following example.

Example 2 We consider a network with 7 players and a transition between $g$ and $g^{\prime}$, as described on Figure 3.
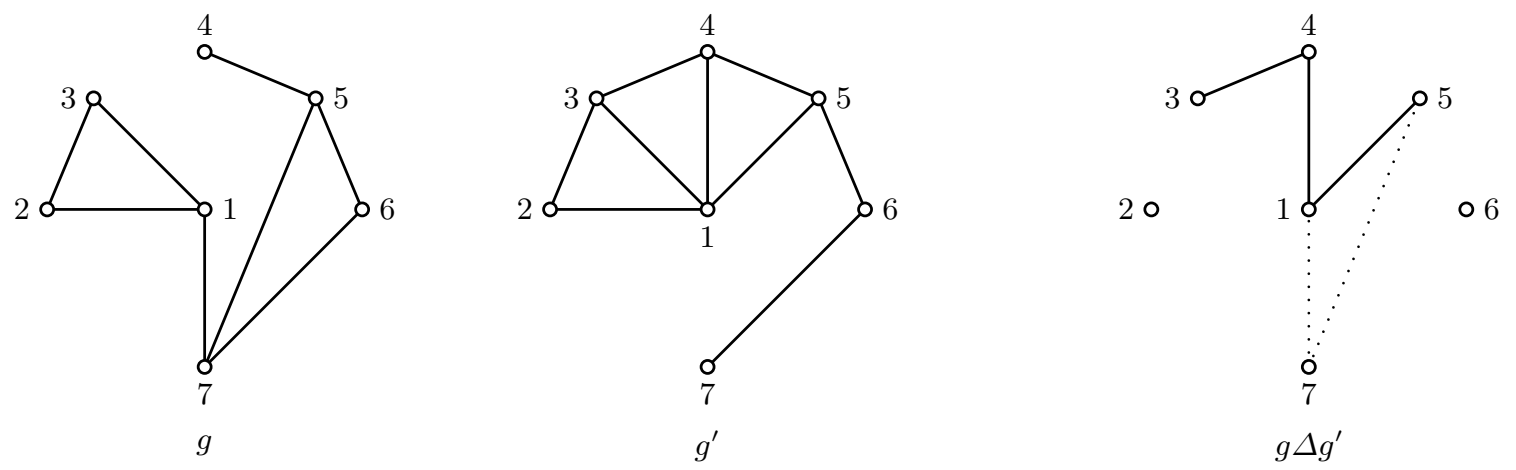

Fig. 3. Example of a transition $g \rightarrow g^{\prime}$. Left: The two networks $g, g^{\prime}$. Right: The graph $g \Delta g^{\prime}$. Entering links are in solid lines, and leaving links are in dotted lines. Players 2 and 6 are inactive, and by (IP) receive 0.

First, we notice that players 2 and 6 are not adjacent to any link in $g \Delta g^{\prime}$, i.e., they are inactive players, and therefore receive 0 ((IP) axiom). In the rest of the discussion, we discard them and concentrate on active players. Suppose now that links 34 and 14 are null. Then players 3 and 4 have no links, and by the Degree axiom, we get $\psi_{3}^{g \rightarrow g^{\prime}}(v)=$ $\psi_{4}^{g \rightarrow g^{\prime}}(v)=0$.

Next, suppose that links 14 and 15 are null. Then there is only one entering link (34), players 3 and 4 are not adjacent to leaving links, so that without further assumption on symmetry, (D) implies that $\psi_{3}^{g \rightarrow g^{\prime}}(v)=\psi_{4}^{g \rightarrow g^{\prime}}(v)$. If moreover, 17 and 57 and symmetric, axiom (D) can be applied to players 1,5 and 7:

$$
2 \psi_{1}^{g \rightarrow g^{\prime}}(v)=2 \psi_{5}^{g \rightarrow g^{\prime}}(v)=\psi_{7}^{g \rightarrow g^{\prime}}(v) .
$$

Lastly, suppose there is no null link. If links 34, 14 and 15 are pairwise symmetric, if links 17 and 57 are symmetric, and if 34 and 17 are antisymmetric (and therefore by Remark 2, any pair of entering and leaving links are antisymmetric), we deduce by (ASEL) that

$$
\psi_{1}^{g \rightarrow g^{\prime}}(v)=3 \psi_{3}^{g \rightarrow g^{\prime}}(v)=\frac{3}{2} \psi_{4}^{g \rightarrow g^{\prime}}(v)=\frac{3}{2} \psi_{5}^{g \rightarrow g^{\prime}}(v)=\frac{3}{2} \psi_{7}^{g \rightarrow g^{\prime}}(v) .
$$

Proposition 2 The LBD scenario allocation rule satisfies $(C),(E),(L),(I P),(D)$ and (ASEL). 
For the proof, see Appendix A.

Theorem 1 The LBD scenario allocation rule is the unique allocation rule satisfying $(C),(E),(L),(I P),(D)$ and $(A S E L)$.

For the proof, we use the following lemma.

Lemma 1 Consider the unanimity game $u_{h}, h \neq \emptyset$. Then the following holds:

(i) A link ij is nonnull for $u_{h}$ if and only if ij $\in h$;

(ii) A pair of links ij, $k l$ is symmetric for $u_{h}$ if and only if both $i j, k l \in h$ or both ij, $k l \notin h$.

Proof: (i) Take $i j \in h$. Then $i j$ is nonnull since $u_{h}(h) \neq u_{h}(h-i j)$. Conversely, take $i j \notin h$. Then $u_{h}(g)=u_{h}(g-i j)$ for every $g$.

(ii) Assume $|h| \geq 2$ and consider $i j, k l \in h$ two distinct links. Then for any $g$ not containing them, $u_{h}(g+i j)=u_{h}(g+k l)=0$. Now assume $\left|g^{N} \backslash h\right| \geq 2$ and consider $i j, k l \notin h$ two distinct links. Then for any $g$ not containing them, $u_{h}(g+i j)=u_{h}(g+k l)=$ $u_{h}(g)$. Conversely, for $h \neq g^{N}$, take $i j \in h$ and $k l \notin h$. Then $1=u_{h}(h-i j+i j) \neq$ $u_{h}(h-i j+k l)=0$, hence these links are not symmetric.

For the proof of Theorem 1, see Appendix A. The independence of the axioms is shown in Appendix B.

\section{Related literature}

In this section we present a brief literature overview of different network allocation rules. Also some of the more recent works that will be mentioned below provide (short) surveys on this issue.

In the seminal work by Myerson (1977) a cooperative game with transferable utilities has been supplemented by a network structure which can be seen as the communication lines between players. In the literature that followed his work the terms communication structures and communication games are usually used. Myerson (1977) introduced and characterized an allocation rule, an extension of the Shapley value (Shapley (1953)) to communication games, which is now called the Myerson value (see also, e.g., Myerson (1980), Aumann and Myerson (1988)). Meessen (1988) proposed an alternative rule for communication situations, called the position value, which has been then characterized by Borm et al. (1992) on the class of communication situations in which the graph is cycle-free. Slikker (2005a,b) provided two characterizations of this value without such restrictions on the graph. Hamiache (1999) presented another rule for communication situations; see also Bilbao et al. (2006). Allocation rules for hypergraph communication situations were studied by van den Nouweland et al. (1992). While Dutta et al. (1998) considered a class of external allocation rules that contains the Myerson value where forming communication links is costless, Slikker and van den Nouweland (2000b) used a natural extension of the Myerson value to determine the payoffs to the players in communication situations with costs for establishing links.

Jackson and Wolinsky (1996) introduced a class of games (called network games) where the value generated depends directly on the network structure, and showed that the 
Myerson value has a direct extension from communication games to network games. They defined the egalitarian allocation rule and the component-wise egalitarian allocation rule. Slikker (2007) characterized axiomatically the Myerson value, the position value and the component-wise egalitarian solution, and also proposed three non-cooperative bargaining procedures that result in the same payoffs as the three rules. For other works concerning non-cooperative foundations of allocation rules, we should mention, e.g., Pérez-Castrillo and Wettstein (2001) who presented an implementation of the Shapley value, and PérezCastrillo and Wettstein (2005) who described a mechanism that ends in the Myerson value of the monotonic cover of the value function.

Caulier (2010) considered a rule that provides payoffs to links, where these payoffs are then divided equally over players. He followed Shapley's characterization for this allocation procedure to the links. The weighted Shapley value defined by Kalai and Samet (1987) was generalized to communication situations in Haeringer (1999) and to hierarchical structures in Slikker and van den Nouweland (2000a). van den Nouweland and Slikker (2012) characterized the position value for network situations where no condition on the underlying network is required. Ghintran (2013) generalized the position value by taking into account the negotiation power of players on the allocation of the worth. She characterized this new weighted position value for communication situations with cyclefree networks. For a similar approach, see also, e.g., Haeringer (2006) who considered allocation rules for cooperative games with transferable utilities. Ghintran et al. (2012) generalized the position value defined for the class of deterministic communication situations, to the class of generalized probabilistic communication situations, and provided two characterizations of this allocation rule.

Although there exists a vast literature on modeling interactions by social and economic networks, the issue of network dynamics has still not received enough attention. The main difference between the works mentioned above and our LBD scenario allocation rule lies in the dynamic aspect of our framework: while the allocation rules presented in the literature assume that the network structure is fixed, the LBD scenario allocation rule is defined for dynamic random network formation processes. An approach similar to ours but used for coalition processes is applied in Faigle and Grabisch $(2012,2013)$.

Examples of the dynamic approach to interactions are presented, e.g., in some extensions of the Jackson-Wolinsky connections model to a dynamic framework; see e.g., Jackson and Watts (2002), Watts (2001, 2002). Konishi and Ray (2003) considered a dynamic model of coalition formation when players are farsighted. As explained above, the important contribution to the dynamic aspect of network formation can be found in Jackson (2005a). A model of dynamic network formation with farsighted players was studied by Dutta et al. (2005). They defined a concept of equilibrium which takes into account farsighted behavior of players and allows for limited cooperation amongst them. Page et al. (2005) introduced a dynamic framework of network formation and analyzed farsightedly consistent directed networks. They studied the notion of a supernetwork which is a collection of directed networks and represents coalitional preferences and rules governing network formation. Page and Wooders (2009) introduced a model of network formation with a set of feasible networks, player preferences, rules of network formation and a dominance relation on feasible networks. The authors characterized sets of network outcomes that are likely to emerge and persist. Also Herings et al. (2009) addressed the question which networks one might expect to emerge in the long run when players are 
farsighted. They provided a full characterization of unique pairwise farsightedly stable sets of networks. More recent work on a dynamic allocation rule in farsighted network formation is presented in Navarro (2013). Page and Wooders (2007) modeled club structures as bipartite networks and formulated the problem of club formation as a game of network formation. They identified club networks that are stable if players are farsighted, and club networks that are stable if players are myopic. Page and Wooders (2010) formulated club formation with multiple memberships as a noncooperative game of network formation and identified conditions sufficient to guarantee that the game has a potential function. In the domain of strategic network formation, Haller et al. (2007) specifies the conditions under which Nash networks exist in the connection model with two-way flow of information and without information decay. Under incomplete information, Harrison and Muñoz (2015) present a specific coalitional refinement of Nash equilibrium based on global games theory, and Song and van der Schaar (forthcoming) study which network structure can emerge in equilibrium and is stable when the agents do not know in advance the value of linking.

Another branch of the literature closely related in spirit to this paper concerns dynamic cooperative games. Oviedo (2000) investigates the core of a repeated cooperative game, i.e., a repeated game where in each round the agents play a cooperative game. The author shows that the core of a repeated cooperative game contains the core of the original cooperative game. Also Kranich et al. (2005) study the core when cooperation takes place in a dynamic setting and define three different concepts of the core for dynamic TU-games: the classical core, the strong sequential core and the weak sequential core. While Predtetchinski (2007) examines the strong sequential core for stationary cooperative games and proves its non-emptiness under some conditions, Habis and Herings (2010) consider the weak sequential core. Habis and Herings (2011) introduce the concept of a transferable utility game with uncertainty and characterize the weak sequential core in this game. They show the non-emptiness of the weak sequential core under some condition. Habis and Herings (2013) study stochastic bankruptcy games and use the weak sequential core as a solution concept for such games. Lehrer and Scarsini (2013) consider dynamic cooperative games, where the worth of coalitions varies over time according to the history of allocations. They define and characterize the concept of intertemporal core.

Although the common feature of the literature mentioned above and the present paper concerns the dynamic aspects of interactions, to the best of our knowledge no previous study deals with a similar allocation rule for dynamic random network formation processes.

\section{Concluding remarks}

We have considered the dynamic random network formation processes, where links may appear and disappear at any time, and a scenario of the process, i.e., a sequence of networks, is the result of a stochastic process, typically a Markov chain. We have established the characterization of the scenario allocation rule for dynamic network processes based on a set of natural axioms. We have shown that if a monotone game is considered, then the link-based flexible network allocation rule of Jackson (2005a) coincides with our LBD link-based allocation rule associated to the so-called natural process. 
Our framework of dynamic network formation can naturally model situations with the set of active players changing over time, where individuals appear or disappear during the process: some players may become involved in later periods of the network formation process, some others can be "active" all the time, some individuals may appear only for a short period of time and disappear forever, or appear again after some time of "silence", etc. When allocating value generated by the dynamic networks we take into account all players that were ever involved in the dynamic network formation process. Hence, many real-life dynamic interactions with appearing/disappearing actors can naturally be modeled by our framework.

There are several directions for follow-up research on this subject. While we have presented the LBD allocation rule which is "fair" in the sense that it is symmetric, we could consider a weighted version of the rule by introducing additionally weights to players when allocating the value among them. For instance, in our Example 1, a rule to reward researchers' involvement in enhancing cooperation could violate symmetry, by taking into account the individuals' frequency of professional missions or their needs for more sophisticated research equipments. Another extension of the present work could include a strategic version of the framework and an endogenous model of a stochastic network formation process.

\section{Appendix A - Proofs}

\section{Proof of Proposition 2}

(C) is immediate from (5). (IP) and (L) are immediate from (6).

(E): By concatenation, it suffices to prove the result for transitions. Consider an elementary transition $g \rightarrow g^{\prime}$, with link $i j$ added or deleted. Then

$$
\sum_{k \in N} \phi_{k}^{g \rightarrow g^{\prime}}(v)=\phi_{i}^{g \rightarrow g^{\prime}}(v)+\phi_{j}^{g \rightarrow g^{\prime}}(v)=2 \times \frac{1}{2}\left(v\left(g^{\prime}\right)-v(g)\right) .
$$

Now, suppose that the transition is not elementary, with $\eta$ created/deleted links. Along each of the $\eta$ ! paths from $g$ to $g^{\prime}$, efficiency holds, therefore

$$
\sum_{k \in N} \phi_{k}^{g \rightarrow g^{\prime}}=\frac{1}{\eta !} \eta !\left(v\left(g^{\prime}\right)-v(g)\right)=v\left(g^{\prime}\right)-v(g) .
$$

(D): We proceed in two steps.

1. We begin by showing that all null links can be discarded without change: $\phi^{g \rightarrow g^{\prime}}(v)=$ $\phi^{g \rightarrow g^{\prime} \backslash i j}(v)$ if $i j \in g^{\prime} \backslash g$ is null for $v$ (a similar reasoning holds for $i j \in g \backslash g^{\prime}$ ). Take such a link $i j$. We have by (6)

$$
\begin{aligned}
\phi_{i}^{g \rightarrow g^{\prime}}(v)= & \frac{1}{2\left|g \Delta g^{\prime}\right| !} \sum_{\sigma}\left(v\left(g+\varepsilon h_{\sigma}^{i j}\right)-v\left(g+h_{\sigma}^{i j}-\varepsilon i j\right)\right) \\
& +\sum_{i k \in g \Delta g^{\prime}, k \neq j} \frac{1}{2\left|g \Delta g^{\prime}\right| !} \sum_{\sigma}\left(v\left(g+\varepsilon h_{\sigma}^{i k}\right)-v\left(g+\varepsilon h_{\sigma}^{i k}-\varepsilon i k\right)\right)
\end{aligned}
$$


with notation of (6).

Since $i j$ is null, the first term in the above equation vanishes. For the second term, we have

$$
\begin{aligned}
\frac{1}{2\left|g \Delta g^{\prime}\right| !} \sum_{\sigma \text { on } g \Delta g^{\prime}}\left(v\left(g+\varepsilon h_{\sigma}^{i k}\right)-v\left(g+\varepsilon h_{\sigma}^{i k}-\varepsilon i k\right)\right)= \\
\quad \frac{\left|g \Delta g^{\prime}\right|}{2\left|g \Delta g^{\prime}\right| !} \sum_{\sigma^{\prime} \text { on } g \Delta\left(g^{\prime}-i j\right)}\left(v\left(g+\varepsilon h_{\sigma^{\prime}}^{i k}\right)-v\left(g+\varepsilon h_{\sigma^{\prime}}^{i k}-\varepsilon i k\right)\right)
\end{aligned}
$$

(note that $h_{\sigma^{\prime}}^{i k}$ is a set in the sequence $\left\{\lambda_{\sigma^{\prime}(1)}\right\},\left\{\lambda_{\sigma^{\prime}(1)}, \lambda_{\sigma^{\prime}(2)}\right\}, \ldots, g^{\prime} \Delta(g-i j)$ ). In summary we get

$\phi_{i}^{g \rightarrow g^{\prime}}(v)=\frac{1}{2\left|g \Delta\left(g^{\prime}-i j\right)\right| !} \sum_{\sigma \text { on }} \sum_{g \Delta\left(g^{\prime}-i j\right)}\left(v\left(g+\varepsilon h_{\sigma}^{i k}\right)-v\left(g+\varepsilon h_{\sigma}^{i k}-\varepsilon i k\right)\right)=\phi_{i}^{g \rightarrow g^{\prime}-i j}$.

2. By Step 1, we may suppose now that no null link exists in $g \Delta g^{\prime}$. We proceed with $g^{\prime} \backslash g$ and players $i$ not adjacent to leaving links, the case of $g \backslash g^{\prime}$ being much that same. If $g^{\prime} \backslash g=\emptyset$, then $i$ becomes inactive and by (6) receive 0 . We suppose then $\left|g^{\prime} \backslash g\right| \geq 1$, with all links in $g^{\prime} \backslash g$ being symmetric, if there are at least two. By symmetry we can set $\nu_{0}(h)=v(g-h), \nu_{1}(h)=v(g-h+i j), \nu_{2}(h)=v(g-h+i j+k l), \ldots, \nu_{\left|g^{\prime} \backslash g\right|}(h)=v\left(g^{\prime}-h\right)$, with $i j, k l, \ldots \in g^{\prime} \backslash g$, and $h$ a set of links in $g \backslash g^{\prime}$. Then for any $i$ such that $i \rightarrow g^{\prime} \backslash g$ and $i \neq g \backslash g^{\prime}$, we get

$$
\begin{aligned}
\phi_{i}^{g \rightarrow g^{\prime}}(v) & =\frac{1}{2\left|g \Delta g^{\prime}\right| !} \sum_{\sigma} \sum_{i k \in g^{\prime} \backslash g}\left(v\left(g+\varepsilon h_{\sigma}^{i k}\right)-v\left(g+\varepsilon h_{\sigma}^{i k}-i k\right)\right) \\
& =\frac{1}{2\left|g \Delta g^{\prime}\right| !} \sum_{i k \in g^{\prime} \backslash g}\left(\sum_{h^{-} \subseteq g \backslash g^{\prime}} \sum_{h^{+} \subseteq g^{\prime} \backslash g-i k} \sum_{\sigma_{h^{-}, h^{+}}}\left(v\left(g-h^{-}+h^{+}+i k\right)-v\left(g-h^{-}+h^{+}\right)\right)\right) \\
& =\frac{1}{2\left|g \Delta g^{\prime}\right| !} \sum_{i k \in g^{\prime} \backslash g}\left(\sum_{h^{-} \subseteq g \backslash g^{\prime}} \sum_{h^{+} \subseteq g^{\prime} \backslash g-i k} \sum_{\sigma_{h^{-}, h^{+}}}\left(\nu_{\left|h^{+}\right|+1}\left(h^{-}\right)-\nu_{\left|h^{+}\right|}\left(h^{-}\right)\right)\right)
\end{aligned}
$$

where $\sigma_{h^{-}, h^{+}}$is any permutation putting first links in $h^{-} \cup h^{+}$in any order. Observe that

$$
K=\sum_{h^{-} \subseteq g \backslash g^{\prime}} \sum_{h^{+} \subseteq g^{\prime} \backslash g-i k} \sum_{\sigma_{h^{-}, h^{+}}}\left(\nu_{\left|h^{+}\right|+1}\left(h^{-}\right)-\nu_{\left|h^{+}\right|}\left(h^{-}\right)\right)
$$

is constant for every $i k \in g^{\prime} \backslash g$. Therefore

$$
\phi_{i}^{g \rightarrow g^{\prime}}(v)=\frac{\ell_{i}\left(g^{\prime} \backslash g\right)}{2\left|g \Delta g^{\prime}\right| !} K,
$$

the desired result.

(ASEL): Consider that links in $\left(g \backslash g^{\prime}\right)^{*}$ and in $\left(g^{\prime} \backslash g\right)^{*}$ are symmetric for $v$, and that any two links $\lambda \in\left(g \backslash g^{\prime}\right)^{*}, \lambda^{\prime} \in\left(g^{\prime} \backslash g\right)^{*}$ are antisymmetric for $v$. Take $i, j \in N$ such that 
$\left(g \backslash g^{\prime}\right)^{*} \leftarrow i \rightarrow\left(g^{\prime} \backslash g\right)^{*}$, and $\left(g^{\prime} \backslash g\right)^{*} \leftarrow j \rightarrow\left(g \backslash g^{\prime}\right)^{*}$. We have by (6):

$$
\begin{aligned}
\phi_{i}^{g \rightarrow g^{\prime}}(v)= & \frac{1}{2\left|\left(g \Delta g^{\prime}\right)^{*}\right|} \sum_{i k \in\left(g^{\prime} \backslash g\right)^{*}} \sum_{m=0}^{\left|\left(g \Delta g^{\prime}\right)^{*}\right|} \sum_{\substack{h^{-} \subseteq\left(g \backslash g^{\prime}\right)^{*} \\
h^{+} \subseteq\left(g^{\prime} \backslash g\right)^{*}-i k \\
\left|h^{+} \cup h^{-}\right|=m}} m !\left(\left|\left(g \Delta g^{\prime}\right)^{*}\right|-m-1\right) !\left(v\left(g+h^{+}-h^{-}+i k\right)\right. \\
& \left.-v\left(g+h^{+}-h^{-}\right)\right)
\end{aligned}
$$

$\phi_{j}^{g \rightarrow g^{\prime}}(v)=\frac{1}{2\left|\left(g \Delta g^{\prime}\right)^{*}\right|} \sum_{j \ell \in\left(g \backslash g^{\prime}\right)^{*}} \sum_{\substack{m=0 \\ h^{-} \subseteq\left(g \backslash g^{\prime}\right)^{*}-j \ell \\ h^{+} \subseteq\left(g^{\prime} \backslash g\right)^{*} \\\left|h^{+} \cup h^{-}\right|=m}}^{\left|\left(g \Delta g^{\prime}\right)^{*}\right|} m !\left(\left|\left(g \Delta g^{\prime}\right)^{*}\right|-m-1\right) !\left(v\left(g+h^{+}-h^{-}-j \ell\right)\right.$

$$
\left.-v\left(g+h^{+}-h^{-}\right)\right)
$$

which we rewrite simply as

$$
\begin{aligned}
& \phi_{i}^{g \rightarrow g^{\prime}}(v)=\frac{1}{2\left|\left(g \Delta g^{\prime}\right)^{*}\right|} \sum_{i k \in\left(g^{\prime} \backslash g\right)^{*}} \phi_{i, i k}^{g \rightarrow g^{\prime}}(v) \\
& \phi_{j}^{g \rightarrow g^{\prime}}(v)=\frac{1}{2 \mid\left(g \Delta g^{\prime}\right)^{*}} \sum_{j \ell \in\left(g \backslash g^{\prime}\right)^{*}} \phi_{j, j \ell}^{g \rightarrow g^{\prime}}(v) .
\end{aligned}
$$

Let us prove that $\phi_{i, i k}^{g \rightarrow g^{\prime}}(v)=\phi_{j, j \ell}^{g \rightarrow g^{\prime}}(v)$ using the fact that $i k$ and $j \ell$ are antisymmetric for $v$, that is, $v\left(g^{\prime \prime}+i k\right)=v\left(g^{\prime \prime}-j \ell\right)$ for every graph $j \ell \in g^{\prime \prime} \not \supset i k$ :

$$
\begin{aligned}
& \phi_{j, j \ell}^{g \rightarrow g^{\prime}}(v)=\sum_{m=0}^{\left|\left(g \Delta g^{\prime}\right)^{*}\right|} m !\left(\left|\left(g \Delta g^{\prime}\right)^{*}\right|-m-1\right) !\left(\sum_{\substack{h^{-} \subseteq\left(g \backslash g^{\prime}\right)^{*}-j \ell \\
h^{+} \subseteq\left(g^{\prime} \backslash g\right)^{*} \\
h^{+} \ngtr i k \\
\left|h^{+} \cup h^{-}\right|=m}}\left(v\left(g+h^{+}-h^{-}-j \ell\right)-v\left(g+h^{+}-h^{-}\right)\right)\right. \\
& \left.+\sum_{\substack{h^{-} \subseteq\left(g \backslash g^{\prime}\right)^{*}-j \ell \\
h^{+} \subseteq\left(g^{\prime} \backslash g\right)^{*} \\
h^{+} \ni i k \\
h^{+} \cup h^{-} \mid=m}}\left(v\left(g+h^{+}-h^{-}-j \ell\right)-v\left(g+h^{+}-h^{-}\right)\right)\right) \\
& =\sum_{m=0}^{\left|\left(g \Delta g^{\prime}\right)^{*}\right|} m !\left(\left|\left(g \Delta g^{\prime}\right)^{*}\right|-m-1\right) !\left(\sum_{\substack{h^{-} \subseteq\left(g \backslash g^{\prime}\right)^{*}-j \ell \\
h^{+} \subseteq\left(g^{\prime} \backslash g\right)^{*} \\
h^{+} \not i k \\
h^{+} \cup h^{-} \mid=m}}\left(v\left(g+h^{+}+i k-h^{-}\right)-v\left(g+h^{+}-h^{-}\right)\right)\right. \\
& \left.+\sum_{\substack{h^{-} \subseteq\left(g \backslash g^{\prime}\right)^{*}-j \ell \\
h^{+} \subseteq\left(g^{\prime} \backslash g\right)^{*} \\
h^{+} \ni i k \\
\left|h^{+} \cup h^{-}\right|=m}}\left(v\left(g+h^{+}-h^{-}-j \ell\right)-v\left(g+h^{+}-i k-h^{-}-j \ell\right)\right)\right) .
\end{aligned}
$$


After a slight rewriting of $\phi_{i, i k}^{g \rightarrow g^{\prime}}(v)$ :

$$
\begin{aligned}
& \phi_{i, i k}^{g \rightarrow g^{\prime}}(v)=\sum_{m=0}^{\left|\left(g \Delta g^{\prime}\right)^{*}\right|} m !\left(\left|\left(g \Delta g^{\prime}\right)^{*}\right|-m-1\right) !\left(\sum_{\substack{h^{-} \subseteq\left(g \backslash g^{\prime}\right)^{*} \\
h^{+} \subseteq\left(g^{\prime} \backslash g\right)^{*}-i k \\
h^{-} \not j \ell \\
\left|h^{+} \cup h^{-}\right|=m}}\left(v\left(g+h^{+}-h^{-}+i k\right)-v\left(g+h^{+}-h^{-}\right)\right)\right. \\
& \left.+\sum_{h^{-} \subseteq\left(g \backslash g^{\prime}\right)^{*}}\left(v\left(g+h^{+}-h^{-}+i k\right)-v\left(g+h^{+}-h^{-}\right)\right)\right) \\
& h^{+} \subseteq\left(g^{\prime} \backslash g\right)^{*}-i k \\
& \begin{array}{c}
h^{-} \ni j \ell \\
h^{+} \cup h^{-} \mid=m
\end{array} \\
& =\sum_{m=0}^{\left|\left(g \Delta g^{\prime}\right)^{*}\right|} m !\left(\left|\left(g \Delta g^{\prime}\right)^{*}\right|-m-1\right) !\left(\sum_{\substack{h^{-} \subseteq\left(g \backslash g^{\prime}\right)^{*} \\
h^{+} \subseteq\left(g^{\prime} \backslash g\right)^{*}-i k \\
h-\not \supset j \ell \\
h^{+} \cup h^{-} \mid=m}}\left(v\left(g+h^{+}+i k-h^{-}\right)-v\left(g+h^{+}-h^{-}\right)\right)\right. \\
& \left.+\sum_{\substack{h^{-} \subseteq\left(g \backslash g^{\prime}\right)^{*}-j \ell \\
h^{+} \subseteq\left(g^{\prime} \backslash g\right)^{*} \\
h^{+} \ni i k \\
\left|h^{+} \cup h^{-}\right|=m}}\left(v\left(g+h^{+}-h^{-}-j \ell\right)-v\left(g+h^{+}-i k-h^{-}-j \ell\right)\right)\right)
\end{aligned}
$$

we see that $\phi_{i, i k}^{g \rightarrow g^{\prime}}(v)=\phi_{j, j \ell}^{g \rightarrow g^{\prime}}(v)$, as desired. Now, by symmetry, $\phi_{i, i k}^{g \rightarrow g^{\prime}}(v)=\phi_{i, i k^{\prime}}^{g \rightarrow g^{\prime}}(v)$ for every two links $i k, i k^{\prime} \in\left(g^{\prime} \backslash g\right)^{*}$, and similarly for $\phi_{j, j \ell}^{g \rightarrow g^{\prime}}(v)$, which proves the result.

We address now the case where a node $i$ is adjacent to links both in $\left(g^{\prime} \backslash g\right)^{*}$ and $\left(g \backslash g^{\prime}\right)^{*}$. In this case, we have:

$$
\begin{aligned}
\phi_{i}^{g \rightarrow g^{\prime}}(v)= & \frac{1}{2\left|\left(g \Delta g^{\prime}\right)^{*}\right|}\left(\sum _ { i k \in ( g ^ { \prime } \backslash g ) ^ { * } } \sum _ { m = 0 } ^ { | ( g \Delta g ^ { \prime } ) ^ { * } | } \sum _ { \begin{array} { c } 
{ h ^ { - } \subseteq ( g \backslash g ^ { \prime } ) ^ { * } } \\
{ h ^ { + } \subseteq ( g ^ { \prime } \backslash g ) ^ { * } - i k } \\
{ | h ^ { + } \cup h ^ { - } | = m }
\end{array} } m ! ( | ( g \Delta g ^ { \prime } ) ^ { * } | - m - 1 ) ! \left(v\left(g+h^{+}-h^{-}+i k\right)\right.\right. \\
& \left.-v\left(g+h^{+}-h^{-}\right)\right) \\
& +\sum_{i \ell \in\left(g \backslash g^{\prime}\right)^{*}} \sum_{m=0}^{\left|\left(g \Delta g^{\prime}\right)^{*}\right|} \sum_{\substack{h^{-} \subseteq\left(g \backslash g^{\prime}\right)^{*}-i \ell \\
h^{+} \subseteq\left(g^{\prime} \backslash g\right)^{*} \\
\left|h^{+} \cup h^{-}\right|=m}} m !\left(\left|\left(g \Delta g^{\prime}\right)^{*}\right|-m-1\right) !\left(v\left(g+h^{+}-h^{-}-i \ell\right)\right. \\
& \left.\left.-v\left(g+h^{+}-h^{-}\right)\right)\right)=\frac{1}{2\left|\left(g \Delta g^{\prime}\right)^{*}\right|}\left(\sum_{i k \in\left(g^{\prime} \backslash g\right)^{*}} \phi_{i, i k}^{g \rightarrow g^{\prime}}(v)+\sum_{i \ell \in\left(g \backslash g^{\prime}\right)^{*}} \phi_{i, i \ell}^{g \rightarrow g^{\prime}}(v)\right) .
\end{aligned}
$$

Proceeding exactly as above shows that $\phi_{i, i k}^{g \rightarrow g^{\prime}}(v)=\phi_{i, i \ell}^{g \rightarrow g^{\prime}}(v)$. Now, by symmetry of all links in $\left(g^{\prime} \backslash g\right)^{*}, \phi_{i, i k}^{g \rightarrow g^{\prime}}(v)=\phi_{i, i k^{\prime}}^{g \rightarrow g^{\prime}}(v)$ for any $i k, i k^{\prime} \in\left(g^{\prime} \backslash g\right)^{*}$, and similarly for links in $\left(g \backslash g^{\prime}\right)^{*}$, which proves the result. 


\section{Proof of Theorem 1}

We begin by proving two technical lemmas characterizing symmetric and antisymmetric links in terms of the Möbius transform of $v$.

Lemma 2 Distinct links $\lambda, \lambda^{\prime}$ are symmetric for $v$ if and only if they are symmetric for $m^{v}$ :

$$
m^{v}(g+\lambda)=m^{v}\left(g+\lambda^{\prime}\right), \quad \forall g \not \supset \lambda, \lambda^{\prime} .
$$

Proof: $\lambda, \lambda^{\prime}$ are symmetric for $v$ if $v(g+\lambda)=v\left(g+\lambda^{\prime}\right)$ for all $g \not \supset \lambda, \lambda^{\prime}$, which is equivalent to

$$
0=\sum_{h \subseteq g+\lambda} m^{v}(h)-\sum_{h \subseteq g+\lambda^{\prime}} m^{v}(h)=\sum_{h \subseteq g}\left(m^{v}(h+\lambda)-m^{v}\left(h+\lambda^{\prime}\right)\right), \quad \forall g \not \supset \lambda, \lambda^{\prime} .
$$

For $g=g^{\emptyset}$ this gives $m^{v}(\lambda)=m^{v}\left(\lambda^{\prime}\right)$. For $g=\left\{\lambda^{\prime \prime}\right\}$ we obtain $m^{v}\left(\lambda^{\prime \prime}+\lambda\right)=m^{v}\left(\lambda^{\prime \prime}+\lambda^{\prime}\right)$. Continuing the process we find the desired result.

We can prove similarly:

Lemma 3 Distinct links $\lambda, \lambda^{\prime}$ are antisymmetric for $v$ if and only if

$$
m^{v}\left(g+\lambda+\lambda^{\prime}\right)=-m^{v}(g+\lambda)-m^{v}\left(g+\lambda^{\prime}\right), \quad \forall g \not \ngtr \lambda, \lambda^{\prime},
$$

where $m^{v}$ is the Möbius inverse of $v$ (see (1)).

We know by Proposition 2 that the LBD rule satisfies all these axioms. It remains to show uniqueness. By (L) and (C), it suffices to prove that for any unanimity game $u_{h}$, any transition $g \rightarrow g^{\prime}, \psi^{g \rightarrow g^{\prime}}\left(u_{h}\right)$ is uniquely determined.

We consider the unanimity game $u_{h}, h \subseteq g^{N}$. Inactive players are those not adjacent to $g \Delta g^{\prime}$ and receive 0 by (IP). Now, by Lemma 1 , links not in $h$ are null. Therefore, if an active player is not adjacent to a link both in $h$ and in $g \Delta g^{\prime}$, by (D) he receives 0 . Then by (E), (D) and (IP) we have in any situation:

$$
u_{h}\left(g^{\prime}\right)-u_{h}(g)=\sum_{i \rightarrow h \cap\left(g \Delta g^{\prime}\right)} \psi_{i}^{g \rightarrow g^{\prime}}\left(u_{h}\right)
$$

and $\psi_{i}^{g \rightarrow g^{\prime}}\left(u_{h}\right)=0$ if $i \not h \cap\left(g \Delta g^{\prime}\right)$.

1. We suppose $g \subseteq g^{\prime}$. From (9), we have:

(i) If $h \cap\left(g^{\prime} \backslash g\right)=\emptyset, \psi_{i}^{g \rightarrow g^{\prime}}\left(u_{h}\right)=0$ for all $i$;

(ii) If $h \cap\left(g^{\prime} \backslash g\right)=\{i j\}$ (only one nonnull link), by (D) it follows that

$$
\psi_{i}^{g \rightarrow g^{\prime}}\left(u_{h}\right)=\psi_{j}^{g \rightarrow g^{\prime}}\left(u_{h}\right)=\frac{u_{h}\left(g^{\prime}\right)-u_{h}(g)}{2},
$$

and the other players adjacent to $g^{\prime} \backslash g$ receive 0 ;

(iii) If $\left|h \cap\left(g^{\prime} \backslash g\right)\right|>1$, by Lemma 1, all links in $h \cap\left(g^{\prime} \backslash g\right)$ are symmetric. Hence by (D), we find for any player $i$ adjacent to $\left(g^{\prime} \backslash g\right)^{*}=h \cap\left(g^{\prime} \backslash g\right)$ :

$$
\psi_{i}^{g \rightarrow g^{\prime}}\left(u_{h}\right)=\frac{\ell_{i}\left(h \cap\left(g^{\prime} \backslash g\right)\right)\left(u_{h}\left(g^{\prime}\right)-u_{h}(g)\right)}{\sum_{j \rightarrow h \cap\left(g^{\prime} \backslash g\right)} \ell_{j}\left(h \cap\left(g^{\prime} \backslash g\right)\right)},
$$

and the others receive 0 . 
Finally,

$$
u_{h}\left(g^{\prime}\right)-u_{h}(g)= \begin{cases}1, & \text { if } h \subseteq g^{\prime} \text { and } h \nsubseteq \subseteq g \\ 0, & \text { otherwise. }\end{cases}
$$

Combining the above results and (11), $\psi^{g \rightarrow g^{\prime}}\left(u_{h}\right)$ is uniquely determined.

2. The case $g^{\prime} \subseteq g$ proceeds similarly.

3. We suppose $g \backslash g^{\prime} \neq \emptyset$ and $g^{\prime} \backslash g \neq \emptyset$. Observe that

$$
u_{h}\left(g^{\prime}\right)-u_{h}(g)= \begin{cases}1, & \text { if } h \subseteq g^{\prime} \text { and } h \nsubseteq g \cap g^{\prime} \\ -1, & \text { if } h \subseteq g \text { and } h \nsubseteq g \cap g^{\prime} \\ 0, & \text { otherwise. }\end{cases}
$$

3.1. Suppose $h \subseteq g^{\prime}$ and $h \not \subseteq g \cap g^{\prime}$, hence $h \cap\left(g \Delta g^{\prime}\right) \neq \emptyset$ and $h \cap\left(g \Delta g^{\prime}\right)=h \cap\left(g^{\prime} \backslash g\right)$. Using (9), we can proceed as in case 1 :

(i) If $h \cap\left(g^{\prime} \backslash g\right)=\{i j\}$, by (D) it follows that

$$
\psi_{i}^{g \rightarrow g^{\prime}}\left(u_{h}\right)=\psi_{j}^{g \rightarrow g^{\prime}}\left(u_{h}\right)=\frac{1}{2} .
$$

(ii) If $\left|h \cap\left(g^{\prime} \backslash g\right)\right|>1$, we find for any player $i$ adjacent to $\left(g^{\prime} \backslash g\right)^{*}=h \cap\left(g \Delta g^{\prime}\right)$ :

$$
\psi_{i}^{g \rightarrow g^{\prime}}\left(u_{h}\right)=\frac{\ell_{i}\left(h \cap\left(g \Delta g^{\prime}\right)\right)}{\sum_{j \rightarrow h \cap\left(g \Delta g^{\prime}\right)} \ell_{j}\left(h \cap\left(g \Delta g^{\prime}\right)\right)},
$$

and the others receive 0 .

3.2. The case $h \subseteq g$ and $h \nsubseteq \subseteq g \cap g^{\prime}$ proceeds similarly and yields for any player $i$ adjacent to $\left(g \backslash g^{\prime}\right)^{*}$

$$
\psi_{i}^{g \rightarrow g^{\prime}}\left(u_{h}\right)=-\frac{\ell_{i}\left(h \cap\left(g \Delta g^{\prime}\right)\right)}{\sum_{j \rightarrow h \cap\left(g \Delta g^{\prime}\right)} \ell_{j}\left(h \cap\left(g \Delta g^{\prime}\right)\right)},
$$

and the others receive 0 .

3.3 Suppose $h \subseteq g \cap g^{\prime}$. Then all links in $g \Delta g^{\prime}$ are null, hence

$$
\psi_{i}^{g \rightarrow g^{\prime}}\left(u_{h}\right)=0, \quad \forall i \in N .
$$

3.4 Suppose $h \nsubseteq g$ and $h \nsubseteq g^{\prime}$. Equation (9) becomes:

$$
\sum_{i \rightarrow h \cap\left(g \Delta g^{\prime}\right)} \psi_{i}^{g \rightarrow g^{\prime}}\left(u_{h}\right)=\sum_{\substack{i \rightarrow h \cap\left(g^{\prime} \backslash g\right) \\ \& i \rightarrow h \cap\left(g \backslash g^{\prime}\right)}} \psi_{i}^{g \rightarrow g^{\prime}}\left(u_{h}\right)+\sum_{\substack{i \rightarrow h \cap\left(g \backslash g^{\prime}\right) \\ \& i \rightarrow h \cap\left(g^{\prime} \backslash g\right)}} \psi_{i}^{g \rightarrow g^{\prime}}\left(u_{h}\right)+\sum_{\substack{i \rightarrow h \cap\left(g^{\prime} \backslash g\right) \\ \& i \rightarrow h \cap\left(g \backslash g^{\prime}\right)}} \psi_{i}^{g \rightarrow g^{\prime}}\left(u_{h}\right)=0 .
$$

3.4.1. Suppose $h \cap\left(g^{\prime} \backslash g\right)=\emptyset$. Then (16) reduces to

$$
\sum_{i \rightarrow h \cap\left(g \backslash g^{\prime}\right)} \psi_{i}^{g \rightarrow g^{\prime}}\left(u_{h}\right)=0 .
$$


By Lemma 1(ii), all links in $h \cap\left(g \backslash g^{\prime}\right)$ are symmetric if there are at least two. Then (D) implies $\psi_{i}^{g \rightarrow g^{\prime}}\left(u_{h}\right)=0$ for all $i \rightarrow h \cap\left(g \Delta g^{\prime}\right)$, and by (IP)

$$
\psi_{i}^{g \rightarrow g^{\prime}}\left(u_{h}\right)=0, \quad \forall i \in N .
$$

3.4.2. The case $h \cap\left(g \backslash g^{\prime}\right)=\emptyset$ proceeds similarly, and we also find (17).

3.4.3 Suppose $h \cap\left(g^{\prime} \backslash g\right) \neq \emptyset$ and $h \cap\left(g \backslash g^{\prime}\right) \neq \emptyset$. We proceed by induction on $\left|h \cap\left(g^{\prime} \backslash g\right)\right|$.

We consider first that $h \cap\left(g^{\prime} \backslash g\right)=\{i j\}$ and the game $v=u_{h}-u_{h-i j}$. Applying Lemma 3, it is easy to check that $i j$ and $k l$ are antisymmetric for $v$, for any $k l \in h \cap\left(g \backslash g^{\prime}\right)$. Also, all links in $h \cap\left(g \backslash g^{\prime}\right)$ are symmetric for $v$ when there are at least two links. Then it follows from (ASEL) w.r.t. $v$ that

$$
\frac{\psi_{i}^{g \rightarrow g^{\prime}}(v)}{\ell_{i}\left(h \cap\left(g \Delta g^{\prime}\right)\right)}=\frac{\psi_{j}^{g \rightarrow g^{\prime}}(v)}{\ell_{j}\left(h \cap\left(g \Delta g^{\prime}\right)\right)}=\frac{\psi_{k}^{g \rightarrow g^{\prime}}(v)}{\ell_{k}\left(h \cap\left(g \Delta g^{\prime}\right)\right)}=\frac{\psi_{l}^{g \rightarrow g^{\prime}}(v)}{\ell_{l}\left(h \cap\left(g \Delta g^{\prime}\right)\right)}
$$

which by linearity (L) turns into

$$
\begin{aligned}
\frac{\psi_{i}^{g \rightarrow g^{\prime}}\left(u_{h}\right)-\psi_{i}^{g \rightarrow g^{\prime}}\left(u_{h-i j}\right)}{\ell_{i}\left(h \cap\left(g \Delta g^{\prime}\right)\right)}=\frac{\psi_{j}^{g \rightarrow g^{\prime}}\left(u_{h}\right)-\psi_{j}^{g \rightarrow g^{\prime}}\left(u_{h-i j}\right)}{\ell_{j}\left(h \cap\left(g \Delta g^{\prime}\right)\right)} & =\frac{\psi_{k}^{g \rightarrow g^{\prime}}\left(u_{h}\right)-\psi_{k}^{g \rightarrow g^{\prime}}\left(u_{h-i j}\right)}{\ell_{k}\left(h \cap\left(g \Delta g^{\prime}\right)\right)} \\
& =\frac{\psi_{l}^{g \rightarrow g^{\prime}}\left(u_{h}\right)-\psi_{l}^{g \rightarrow g^{\prime}}\left(u_{h-i j}\right)}{\ell_{l}\left(h \cap\left(g \Delta g^{\prime}\right)\right)} .
\end{aligned}
$$

Since $(h-i j) \cap\left(g^{\prime} \backslash g\right)=\emptyset$, we are back either to case 3.2 (if $h-i j \subseteq g$ ) or case 3.4.1. (otherwise). In the latter case, $\psi^{g \rightarrow g^{\prime}}\left(u_{h-i j}\right)=0$ and we obtain

$$
\frac{\psi_{i}^{g \rightarrow g^{\prime}}\left(u_{h}\right)}{\ell_{i}\left(h \cap\left(g \Delta g^{\prime}\right)\right)}=\frac{\psi_{j}^{g \rightarrow g^{\prime}}\left(u_{h}\right)}{\ell_{j}\left(h \cap\left(g \Delta g^{\prime}\right)\right)}=\frac{\psi_{k}^{g \rightarrow g^{\prime}}\left(u_{h}\right)}{\ell_{k}\left(h \cap\left(g \Delta g^{\prime}\right)\right)}=\frac{\psi_{l}^{g \rightarrow g^{\prime}}\left(u_{h}\right)}{\ell_{l}\left(h \cap\left(g \Delta g^{\prime}\right)\right)} .
$$

In the former case, (14) applies to $k$ and $l$, while $i, j$ receive 0 with $u_{h-i j}$. This yields:

$$
\begin{array}{r}
\frac{\psi_{i}^{g \rightarrow g^{\prime}}\left(u_{h}\right)}{\ell_{i}\left(h \cap\left(g \Delta g^{\prime}\right)\right)}=\frac{\psi_{j}^{g \rightarrow g^{\prime}}\left(u_{h}\right)}{\ell_{j}\left(h \cap\left(g \Delta g^{\prime}\right)\right)}=\frac{\psi_{k}^{g \rightarrow g^{\prime}}\left(u_{h}\right)+\ell_{k}\left((h-i j) \cap\left(g \Delta g^{\prime}\right)\right) / L}{\ell_{k}\left(h \cap\left(g \Delta g^{\prime}\right)\right)} \\
=\frac{\psi_{l}^{g \rightarrow g^{\prime}}\left(u_{h}\right)+\ell_{l}\left((h-i j) \cap\left(g \Delta g^{\prime}\right)\right) / L}{\ell_{l}\left(h \cap\left(g \Delta g^{\prime}\right)\right)}
\end{array}
$$

with $L=\sum_{i^{\prime} \rightarrow(h-i j) \cap\left(g \Delta g^{\prime}\right)} \ell_{i^{\prime}}\left((h-i j) \cap\left(g \Delta g^{\prime}\right)\right)$. Now, consider again equation (16). Observe that $\psi_{i}^{g \rightarrow g^{\prime}}\left(u_{h}\right), \psi_{j}^{g \rightarrow g^{\prime}}\left(u_{h}\right), \psi_{k}^{g \rightarrow g^{\prime}}\left(u_{h}\right)$ and $\psi_{l}^{g \rightarrow g^{\prime}}\left(u_{h}\right)$ are variables in this equation. Moreover, we can apply (D) w.r.t. $u_{h}$ to the nodes $i^{\prime}$ in the second term (those adjacent to $h \cap\left(g \backslash g^{\prime}\right)$ but not to $\left.h \cap\left(g^{\prime} \backslash g\right)\right)$, while the first and third term can only concern $i$ or $j$ or both. It follows that (16) contains only the variables $\psi_{i}^{g \rightarrow g^{\prime}}\left(u_{h}\right), \psi_{j}^{g \rightarrow g^{\prime}}\left(u_{h}\right), \psi_{k}^{g \rightarrow g^{\prime}}\left(u_{h}\right)$ and $\psi_{l}^{g \rightarrow g^{\prime}}\left(u_{h}\right)$. Substituting into it the different equalities in (18) or (19) determine these variables uniquely. Note that in the case of (18), all active players receive 0 .

Suppose now that $\psi_{i}^{g \rightarrow g^{\prime}}\left(u_{h}\right)$ is known till $\left|h \cap\left(g^{\prime} \backslash g\right)\right|=m<\left|g^{\prime} \backslash g\right|$ and let us determine $\psi^{g \rightarrow g^{\prime}}\left(u_{h}\right)$ when $\left|h \cap\left(g^{\prime} \backslash g\right)\right|=m+1$. Consider the game

$$
v=\sum_{h^{\prime} \subseteq h \cap\left(g^{\prime} \backslash g\right)}(-1)^{\left|h^{\prime}\right|} u_{h \backslash h^{\prime}}
$$


We claim that any $i j \in h \cap\left(g^{\prime} \backslash g\right)$ and any $k \ell \in h \cap\left(g \backslash g^{\prime}\right)$ are antisymmetric for $v$, and that moreover all $i j \in h \cap\left(g^{\prime} \backslash g\right)$ are symmetric for $v$, and so are all links in $h \cap\left(g \backslash g^{\prime}\right)$.

Proof of The Claim: The values of the Möbius inverse of $v$ are 1 for $h,-1$ for $h-i j, 1$ for $h-i j-i^{\prime} j^{\prime},-1$ for $h-i j-i^{\prime} j^{\prime}-i^{\prime \prime} j^{\prime \prime}$, etc., with $i j, i^{\prime} j^{\prime}, i^{\prime \prime} j^{\prime \prime} \in h \cap\left(g^{\prime} \backslash g\right)$, and 0 otherwise. Let us check antisymmetry for $i j \in h \cap\left(g^{\prime} \backslash g\right)$ and $k \ell \in h \cap\left(g \backslash g^{\prime}\right)$ by Lemma 3. We must check that

$$
m^{v}(g+i j+k \ell)=-m^{v}(g+i j)-m^{v}(g+k \ell), \quad \forall g \not \supset i j, k \ell .
$$

Observe that $m^{v}(g+i j)=0$ for every $g \not \supset i j, k \ell$. By construction either $m^{v}(g+$ $i j+k l)$ and $m^{v}(g+k \ell)$ are both 0 (if $g \neq h-h^{\prime}-k \ell$ for some $h^{\prime} \subseteq h \cap\left(g^{\prime} \backslash g\right)$ containing $i j)$, or $m^{v}(g+i j+k \ell)=-m^{v}(g+k \ell)$ holds. In both cases, the equality holds. Now, by Lemma 2, symmetry holds for any two links in $h \cap\left(g^{\prime} \backslash g\right)$ if $m^{v}$ is symmetric for these links, which is the case by construction. Finally, any two links in $h \cap\left(g \backslash g^{\prime}\right)$ are symmetric for $v$ because they are symmetric for each $u_{h \backslash h^{\prime}}$, $h^{\prime} \subseteq h \cap\left(g \backslash g^{\prime}\right)$.

Hence (ASEL) w.r.t. $v$ can be applied to any pair of links $i j \in h \cap\left(g^{\prime} \backslash g\right)$ and $k \ell \in h \cap\left(g \backslash g^{\prime}\right)$, and since by induction hypothesis, all $\psi^{g \rightarrow g^{\prime}}\left(u_{h \backslash h^{\prime}}\right), h^{\prime} \neq \emptyset$ are determined, it follows that (18) holds, for any $i j \in h \cap\left(g^{\prime} \backslash g\right)$ and any $k \ell \in h \cap\left(g \backslash g^{\prime}\right)$. Finally, the successive substitutions into (16) determine $\psi^{g \rightarrow g^{\prime}}\left(u_{h}\right)$ uniquely.

\section{Appendix B - Independence of the axioms}

We first easily establish the independence of (C), (L) with the other axioms. Indeed, defining for a scenario $\mathcal{G}=g_{0}, g_{1}, \ldots, g_{q}$ the following value

$$
\psi^{\mathcal{G}}(v)=f\left(\phi^{g_{0} \rightarrow g_{1}}(v), \phi^{g_{1} \rightarrow g_{2}}(v), \ldots, \phi^{g_{q-1} \rightarrow g_{q}}(v)\right),
$$

where $f$ is an operator different from the sum, satisfies all axioms but (C). Similarly, defining for a scenario $\mathcal{G}=g_{0}, g_{1}, \ldots, g_{q}$ the value

$$
\psi^{\mathcal{G}}(v)=\sum_{t=1}^{q-1}\left(\oplus_{h \subseteq g^{N}} m^{v}(h) \phi^{g_{t} \rightarrow g_{t+1}}\left(u_{h}\right)\right)
$$

with $v=\sum_{h \subseteq g^{N}} m^{v}(h) u_{h}$ and $\oplus$ is an operator different from the sum, satisfies all axioms but (L).

It remains to show that (E), (IP), (D) and (ASEL) are independent for the axiomatization of $\phi^{g \rightarrow g^{\prime}}\left(u_{h}\right)$, for any transition $g \rightarrow g^{\prime}$ and any unanimity game $u_{h}$.

(i) Axiom (E): Removing the normalization constant $\frac{1}{2\left|g \Delta g^{\prime}\right| !}$ in (6) yields a allocation rule satisfying all axioms except $(\mathrm{E})$.

(ii) Axiom (IP): Define $\psi^{g \rightarrow g^{\prime}}=\phi^{g \rightarrow g^{\prime}}$ if $1 \rightarrow g \Delta g^{\prime}$, and if not (1 is inactive):

$$
\psi_{i}^{g \rightarrow g^{\prime}}= \begin{cases}(1-\alpha)\left(v\left(g^{\prime}\right)-v(g)\right), & \text { if } i=1 \\ \frac{\alpha}{2\left|g \Delta g^{\prime}\right| !} \sum_{\sigma} \sum_{i k \in g \Delta g^{\prime}}\left(v\left(g+\varepsilon h_{\sigma}^{i k}\right)-v\left(g+\varepsilon h_{\sigma}^{i k}-\varepsilon i k\right)\right), & \text { if } i \rightarrow g \Delta g^{\prime} \\ 0, & \text { otherwise }\end{cases}
$$


with same notation as in (6) and for some fixed positive $\alpha$. It can be checked that (IP) is not satisfied, but (E), (D) and (ASEL) are (the two last are satisfied because either $\phi^{g \rightarrow g^{\prime}}$ is used, or only the 2nd and 3d lines in the above formula).

(iii) Axiom (D): Define $\psi^{g \rightarrow g^{\prime}}$ to be identical to $\phi^{g \rightarrow g^{\prime}}$, except when $\left(g \Delta g^{\prime}\right)^{*}$ reduces to one link, say $i j$, in which case:

$$
\psi_{i}^{g \rightarrow g^{\prime}}(v)=\frac{1}{3}\left(v\left(g^{\prime}\right)-v(g)\right), \quad \psi_{j}^{g \rightarrow g^{\prime}}(v)=\frac{2}{3}\left(v\left(g^{\prime}\right)-v(g)\right) .
$$

Then (IP), (E) and (ASEL) are satisfied but not (D).

(iv) Axiom (ASEL): Observe that (ASEL) is only used in case 3.4.1 in the proof of Theorem 1 , that is, for the game $u_{h}$ with $h \cap\left(g \backslash g^{\prime}\right) \neq \emptyset$ and $h \cap\left(g^{\prime} \backslash g\right) \neq \emptyset$. It suffices then to take $\psi_{i}^{g \rightarrow g^{\prime}}\left(u_{h}\right)=0$ for all active players, i.e., those adjacent to $h \cap\left(g \Delta g^{\prime}\right)$. Then (16), which is imposed by (IP), (E) and (D), is satisfied, but not (19), which is imposed by (ASEL). 


\section{Bibliography}

R. Aumann and R. Myerson. Endogenous formation of links between players and coalitions: an application of the Shapley value. In A. Roth, editor, The Shapley Value, pages 175-191. Cambridge University Press, 1988.

J. Bilbao, N. Jiménez, and J. López. A note on a value with incomplete communication. Games and Economic Behavior, 54(2):419-429, 2006.

P. Borm, G. Owen, and S. Tijs. On the position value for communication situations. SIAM Journal on Discrete Mathematics, 5:305-320, 1992.

J.-F. Caulier. Contribution to Collective Choice Theory. Académie Universitaire Louvain, Belgium, 2010. Ph.D. Thesis, Facultés Universitaires Saint Louis.

B. Dutta and M. O. Jackson. Networks and Groups: Models of Strategic Formation. Springer-Verlag, Heidelberg, 2003.

B. Dutta, A. van den Nouweland, and S. Tijs. Link formation in cooperative situations. International Journal of Game Theory, 27:245-256, 1998.

B. Dutta, S. Ghosal, and D. Ray. Farsighted network formation. Journal of Economic Theory, 122(2):143-164, 2005.

U. Faigle and M. Grabisch. Values for Markovian coalition processes. Economic Theory, 51:505-538, 2012.

U. Faigle and M. Grabisch. A note on values for Markovian coalition processes. Economic Theory Bulletin, 1:111-122, 2013.

A. Ghintran. Weighted position values. Mathematical Social Sciences, 65:157-163, 2013.

A. Ghintran, E. González-Aranguena, and C. Manuel. A probabilistic position value. Annals of Operations Research, 201:183-196, 2012.

H. Habis and P. J. J. Herings. A note on the weak sequential core of dynamic TU games. International Game Theory Review, 12:407-416, 2010.

H. Habis and P. J. J. Herings. Transferable utility games with uncertainty. Journal of Economic Theory, 146:2126-2139, 2011.

H. Habis and P. J. J. Herings. Stochastic bankruptcy games. International Journal of Game Theory, 42:973-988, 2013.

G. Haeringer. Weighted Myerson value. International Game Theory Review, 1(2):187$192,1999$.

G. Haeringer. A new weight scheme for the Shapley value. Mathematical Social Sciences, 52(1):88-98, 2006.

H. Haller, J. Kamphorst, and S. Sarangi. (Non-)existence and scope of Nash networks. Economic Theory, 31:597-604, 2007.

G. Hamiache. A value with incomplete communication. Games and Economic Behavior, 26(1):59-78, 1999.

R. Harrison and R. Muñoz. Stability and equilibrium selection in a link formation game. Economic Theory, 37:335-345, 2015.

P. J.-J. Herings, A. Mauleon, and V. Vannetelbosch. Farsightedly stable networks. Games and Economic Behavior, 67:526-541, 2009.

M. O. Jackson. Allocation rules for network games. Games and Economic Behavior, 51: 128-154, 2005a. 
M. O. Jackson. A survey of network formation models: stability and efficiency. In G. Demange and M. Wooders, editors, Group Formation in Economics: Networks, Clubs, and Coalitions, pages 11-49. Cambridge University Press, Cambridge, UK, 2005b.

M. O. Jackson. Social and Economic Networks. Princeton University Press, 2008.

M. O. Jackson and A. Watts. The evolution of social and economic networks. Journal of Economic Theory, 106(2):265-295, 2002.

M. O. Jackson and A. Wolinsky. A strategic model of social and economic networks. Journal of Economic Theory, 71:44-74, 1996.

E. Kalai and D. Samet. On weighted Shapley values. International Journal of Game Theory, 16(3):205-222, 1987.

H. Konishi and D. Ray. Coalition formation as a dynamic process. Journal of Economic Theory, 110:1-41, 2003.

L. Kranich, A. Perea, and H. Peters. Core concepts for dynamic TU games. International Game Theory Review, 7:43-61, 2005.

E. Lehrer and M. Scarsini. On the core of dynamic cooperative games. Dynamic Games and Applications, 3:359-373, 2013.

R. Meessen. Communication Games. Master Thesis, University of Nijmegen, The Netherlands, 1988.

R. B. Myerson. Graphs and cooperation in games. Mathematics of Operations Research, 2:225-229, 1977.

R. B. Myerson. Conference structures and fair allocation rules. International Journal of Game Theory, 9(3):169-182, 1980.

N. Navarro. Expected fair allocation in farsighted network formation. Social Choice and Welfare, 43(2):287-308, 2013.

J. Oviedo. The core of a repeated n-person cooperative game. European Journal of Operational Research, 127:519-524, 2000.

F. H. Page and M. Wooders. Networks and clubs. Journal of Economic Behavior and Organization, 64:406-425, 2007.

F. H. Page and M. Wooders. Strategic basins of attraction, the path dominance core, and network formation games. Games and Economic Behavior, 66:462-487, 2009.

F. H. Page and M. Wooders. Club networks with multiple memberships and noncooperative stability. Games and Economic Behavior, 70:12-20, 2010.

F. H. Page, M. Wooders, and S. Kamat. Networks and farsighted stability. Journal of Economic Theory, 120:257-269, 2005.

D. Pérez-Castrillo and D. Wettstein. Bidding for the surplus: a non-cooperative approach to the Shapley value. Journal of Economic Theory, 100(2):274-294, 2001.

D. Pérez-Castrillo and D. Wettstein. Forming efficient networks. Economic Letters, 87(1): 83-87, 2005.

A. Predtetchinski. The strong sequential core for stationary cooperative games. Games and Economic Behavior, 61:50-66, 2007.

L. S. Shapley. A value for n-person games. In H. W. Kuhn and A. W. Tucker, editors, Annals of Mathematics Studies, 28, pages 307-317. Princeton University Press, 1953.

M. Slikker. A characterization of the position value. International Journal of Game Theory, 33(4):505-514, 2005a.

M. Slikker. Link monotonic allocation schemes. International Game Theory Review, 7(4): 473-489, 2005b. 
M. Slikker. Bidding for surplus in network allocation problems. Journal of Economic Theory, 137:493-511, 2007.

M. Slikker and A. van den Nouweland. Communication situations with asymmetric players. Mathematical Methods of Operations Research, 52:39-56, 2000a.

M. Slikker and A. van den Nouweland. Network formation with costs for establishing links. Review of Economic Design, 5(3):333-362, 2000b.

M. Slikker and A. van den Nouweland. Social and Economic Networks in Cooperative Game Theory. Kluwer Academic Publishers, Boston, 2001.

Y. Song and M. van der Schaar. Dynamic network formation with incomplete information. Economic Theory, forthcoming.

A. van den Nouweland. Models of network formation in cooperative games. In G. Demange and M. Wooders, editors, Group Formation in Economics: Networks, Clubs, and Coalitions. Cambridge University Press, Cambridge, UK, 2005.

A. van den Nouweland and M. Slikker. An axiomatic characterization of the position value for network situations. Mathematical Social Sciences, 64:266-271, 2012.

A. van den Nouweland, P. Borm, and S. Tijs. Allocation rules for hypergraph communication situations. International Journal of Game Theory, 20:255-268, 1992.

A. Watts. A dynamic model of network formation. Games and Economic Behavior, 34: 331-341, 2001.

A. Watts. Non-myopic formation of circle networks. Economic Letters, 74:277-282, 2002. 